\title{
The potential of future aircraft technology for noise and pollutant emissions reduction
}

\author{
W R Graham ${ }^{1}$, C A Hall, M Vera Morales ${ }^{2}$ \\ Institute for Aviation and the Environment, University of Cambridge, Cambridge, CB3 ODY, UK
}

\begin{abstract}
The negative external impacts of aviation are currently under unprecedented scrutiny. In response, a number of studies into future prospects for improvement have recently been carried out. This paper reviews these studies and discusses their combined implications for emissions of carbon dioxide, oxides of nitrogen, and noise. The results are also compared with targets for emissions reduction proposed by ACARE and NASA. It is concluded that significant future gains are achievable, but not to the extent implied by the ACARE and NASA targets, which represent an unrealistically optimistic view of technological potential over the next 20-40 years. The focus on technological advance also deflects attention from the substantial benefits available from combining present-day technology with behavioural change. Finally, difficult policy decisions will be necessary; the greatest benefits are associated with technological developments that will require major, and long-term, investment for their realisation, and there will be increasing conflict between environmental and noise goals.
\end{abstract}

Keywords: climate change, aircraft pollution, aircraft noise

$\begin{array}{ll} & \\ & \\ \text { ACARE } & \text { Advisory Council for Aviation Research and Innovation in Europe } \\ \text { BPR } & \text { By-pass ratio of a turbofan engine } \\ \text { BWB } & \text { Blended wing-body aircraft configuration } \\ \text { CAEP } & \text { Committee on Aviation Environmental Protection } \\ \text { CO }_{2} & \text { Carbon dioxide } \\ \text { CRC } & \text { Conceptual Research Corporation (NASA N+3 contractor) } \\ \text { EIS } & \text { Entry into service } \\ \text { EPNdB } & \text { Effective perceived noise level in decibels } \\ \text { GBD } & \text { Greener by Design (Royal Aeronautical Society) } \\ \text { HLFC } & \text { Hybrid laminar flow control } \\ \text { ICAO } & \text { International Civil Aviation Organisation } \\ \text { ICR } & \text { Inter-cooled recuperative engine thermodynamic cycle } \\ \text { LFW } & \text { Laminar flying wing aircraft configuration } \\ \text { MIT } & \text { Massachusetts Institute of Technology (NASA N+3 contractor) } \\ \text { NACRE } & \text { New Aircraft Concepts Research (EU project) } \\ \text { NASA } & \text { National Aeronautics and Space Administration } \\ \text { NLF } & \text { Natural laminar flow } \\ \text { NOx } & \text { Oxides of nitrogen } \\ \text { SAI } & \text { Silent Aircraft Initiative (Cambridge-MIT Institute project) } \\ \text { TW } & \text { Conventional, 'tube and wing', aircraft configuration } \\ & \end{array}$

\section{Introduction}

The introduction of jet-propelled passenger transport aircraft fifty-five years ago ushered in an era of unprecedented human mobility. Equally, it was associated with noise and local air quality issues that were painfully obvious to those living near airports. Today, these aircraft emissions are regulated, with benefits that are immediately evident to the naked eye and ear when vehicles from the two eras are compared directly. Unfortunately, however, much of this improvement is offset by the huge increase in air traffic over the

\footnotetext{
${ }^{1}$ Corresponding author: wrg11@cam.ac.uk, +44 1223332634.

2 Present address: Department of Aeronautical and Automotive Engineering, Loughborough University, Leicestershire, LE11 3TU.
} 
intervening period. As a result, pressure to reduce noise and local chemical pollutants (specifically oxides of nitrogen, or 'NOx') remains high.

In addition, early jet engines were extremely inefficient; they displaced propellors nonetheless because of their ability to deliver thrust at high flight speed with low weight. Historically, their efficiency was not seen as an environmental problem, and the only driver for improvements was fuel cost. Now, however, with carbon dioxide $\left(\mathrm{CO}_{2}\right)$ from the combustion of fossil fuel recognised as the dominant source of climate change, there is also societal pressure. As a result, the negative external impacts of mass air travel are under scrutiny as never before.

In 2001, recognising this situation, the Advisory Council for Aviation Research and Innovation in Europe (ACARE) published a 'vision' for 2020 (European Commission, 2001); this set targets of 50\% reductions in fuel-burn and perceived noise, and $80 \%$ in landing/take-off NOx emissions, relative to year-2000 aircraft. With both Airbus' and Boeing's plans to this date now established, it has become clear that these targets will not be achieved. They have been replaced by a new set, 'FlightPath 2050' (European Commission, 2011), which calls for reductions of $75 \%, 65 \%$ and $90 \%$ respectively by 2050 . In the U.S., similar goals have been proposed by NASA for the ' $\mathrm{N}+2$ ' ' (service-entry 2025) and ' $\mathrm{N}+3$ ' (service-entry 2030-35) generations of aircraft (Collier, 2012). These are summarised, along with their ACARE counterparts, in Table 1. (Note that CAEP 6 and Stage 4 are regulatory levels; they are explained in Section 2.) Associated with this activity has been a surge in studies into future mitigation prospects, many of which invoke either radical technology developments or novel aircraft configurations.

Table 1 Fuel-burn and emissions reduction goals put forward by ACARE and NASA

\begin{tabular}{|c|c|c|c|c|}
\hline \multirow{2}{*}{ Category } & \multicolumn{2}{|c|}{ ACARE } & \multicolumn{2}{|c|}{ NASA } \\
\hline & Vision 2020 & FlightPath 2050 & $N+2(2025)$ & $N+3(2030-35)$ \\
\hline \multirow{2}{*}{ Fuel } & $50 \%$ & $75 \%$ & $50 \%$ & $60 \%$ \\
\hline & \multicolumn{2}{|c|}{ Relative to year-2000 aircraft } & \multicolumn{2}{|c|}{ Relative to year-2005 best-in-class } \\
\hline \multirow{2}{*}{ NOx } & $80 \%$ & $90 \%$ & $75 \%$ & $80 \%$ \\
\hline & \multicolumn{2}{|c|}{ Relative to year-2000 aircraft } & \multicolumn{2}{|c|}{ Relative to CAEP 6} \\
\hline \multirow{2}{*}{ Noise } & $50 \%$ & $65 \%$ & $42 \mathrm{EPNdB}$ & $71 \mathrm{EPNdB}$ \\
\hline & \multicolumn{2}{|c|}{ Relative to year-2000 aircraft } & \multicolumn{2}{|c|}{ Cumulative, relative to Stage 4} \\
\hline
\end{tabular}

The time is thus ripe to take stock, and this is the aim of the current paper. In particular, we seek to review the potential of technological advances in the aircraft itself, in the light of ACARE's and NASA's stated goals. At this point, it should be recognised that some contribution towards the fuel-burn and noise targets is envisaged from operational improvements, via elimination of air-traffic-management inefficiencies and alterations to landing approach procedures (see Reynolds, this issue). Aspects of the latter that are relevant to the regulatory noise measures targeted by ACARE and NASA are accounted for in the studies reported here. Efficiency gains in air-traffic management are typically not; however they have progressively less impact as the fuel-burn target becomes more aggressive. (For example, if 5\% of current fuel consumption is due to air-traffic-management inefficiencies, and $60 \%$ reduction is required, the aircraft-alone reduction must be $58 \%$.) We will therefore compare predicted technological benefits directly with the targets.

As a final point, one could question the use of fuel consumption as a metric. Emissions of the associated pollutant, $\mathrm{CO}_{2}$, can also be reduced via the use of alternative fuels (see Hileman, this issue). This issue, however, is outside the scope of the current review.

The structure of the paper is as follows. We first consider the relevant pollutants, and the factors influencing their generation. Then, in Section 3, we describe the studies reviewed here. Section 4 presents a comparative analysis of the studies, in order to identify areas of agreement, and of inconsistency. This then forms the basis for a discussion of future prospects, in Section 5. Our conclusions are summarised in Section 6.

\section{Background}

Aircraft emit a number of pollutants, of which three $-\mathrm{CO}_{2}, \mathrm{NOx}$, and noise - have received most attention to date. This section reviews production mechanisms and historical trends for each in turn.

\section{$2.1 \quad$ Carbon dioxide}

$\mathrm{CO}_{2}$ has only been viewed as a pollutant since its recognition as the dominant greenhouse gas responsible for global warming. For a given fuel type, the amount emitted is directly proportional to the mass of fuel burnt. As 
fuel-burn is a key component of aircraft operating cost, economic considerations have driven significant reductions in aircraft $\mathrm{CO}_{2}$ emissions since the beginning of the jet era. Figure 1 demonstrates these gains, but also shows that the most dramatic improvements were achieved early on. The ACARE and NASA goals of further reductions in excess of 50\% thus require a major departure from straightforward extrapolation of presentday trends.

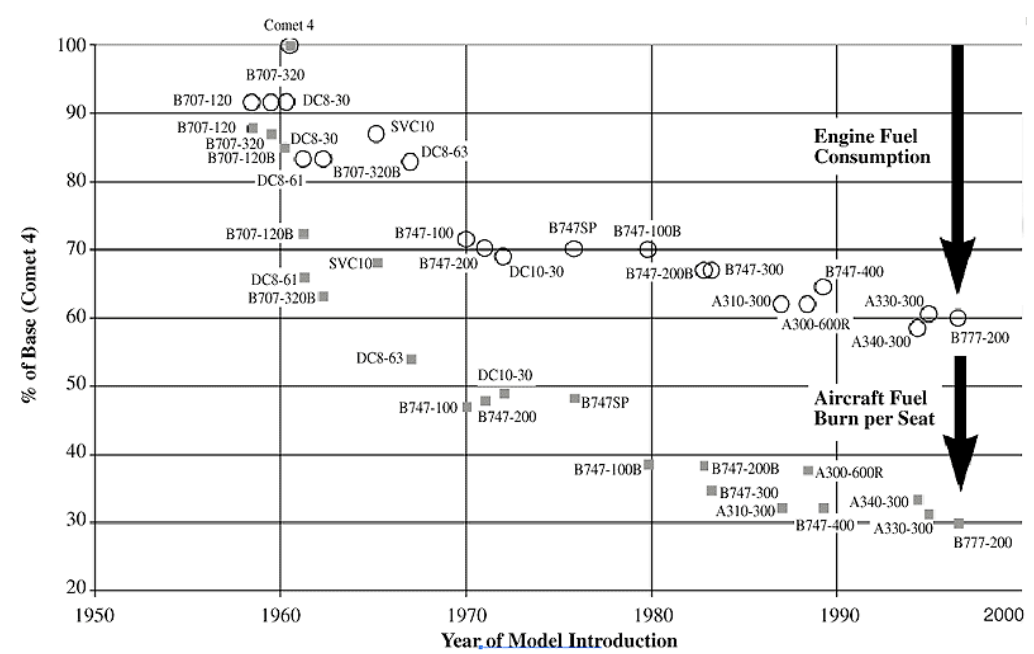

Figure 1 Historical data on aircraft fuel-burn (Henderson and Wickrama, 1999)

To understand how aircraft technology affects fuel-burn, we consider the classical range equation (Torenbeek, 1997). For an idealised cruise, with aircraft operating parameters fixed, it can be arranged to give the following expression for the fuel consumption per payload-range:

$$
\frac{W_{f}}{R W_{p}}=\frac{1}{R}\left(1+\frac{W_{e}}{W_{p}}\right)\left[\exp \left(\frac{R}{H \eta L / D}\right)-1\right],
$$

in which $R$ is the range, $W_{f}$ the weight of the fuel burnt, $W_{p}$ the payload weight, $W_{e}$ the aircraft empty weight, $\eta$ the engine efficiency and $L / D$ the ratio of lift to drag. The parameter $H$ represents the intrinsic energy content of the fuel; for kerosene it takes the value $4,350 \mathrm{~km}$. For a given range and payload, improvements in aircraft aerodynamics, engine performance and structural weight will decrease the amount of fuel burnt per passengerkilometre, through an increase in $L / D$, an increase in $\eta$, and a decrease in $W_{e}$ respectively. As the lift of an aircraft in cruise is equal to its weight, the first of these is equivalent to a reduction in drag. This quantity consists of two components: the zero-lift drag, which is largely due to friction between the aircraft skin and the flow, and the lift-dependent drag, which is dominated by the 'induced drag' associated with wasted kinetic energy in the aircraft's wake. Induced drag depends on the 'aspect ratio' of the wing; it is reduced when the span is increased.

The parametric dependence of Eq. (1) is even clearer if the argument of the exponential is small, in which case it can be simplified to:

$$
\frac{W_{f}}{R W_{p}}=\left(1+\frac{W_{e}}{W_{p}}\right) \frac{1}{H \eta L / D},
$$

independent of the range. This represents an optimal limiting case in which the fuel required to carry the mission fuel becomes negligible. The value given by the exact expression, (1), is always greater than this, and becomes significantly so for greater ranges; long-range aircraft have an inherent tendency to be less efficient than short-range aircraft. For this reason, Green (2002) has proposed that future long-haul air travel should be organised in stages of no longer than 7,500 km (4,050 nmi), using aircraft specifically designed for this distance.

In practice, an aircraft also consumes fuel in reaching its cruising altitude, and this component becomes significant at very short ranges. Its influence can be seen in Fig. 2, which shows results for representative aircraft from the turboprop, regional-jet, narrow-body and wide-body categories (Vera Morales et al., 2011). The turboprop and regional jet only approach their best fuel efficiency for flights in excess of $1,000 \mathrm{~km}(540$ $\mathrm{nmi})$; the narrow- and wide-bodies are significantly compromised over distances below 2,000 km (1,080 $\mathrm{nmi})$. (Note that the aforementioned deterioration with increasing range is not evident here, because it occurs at values greater than those plotted.) 


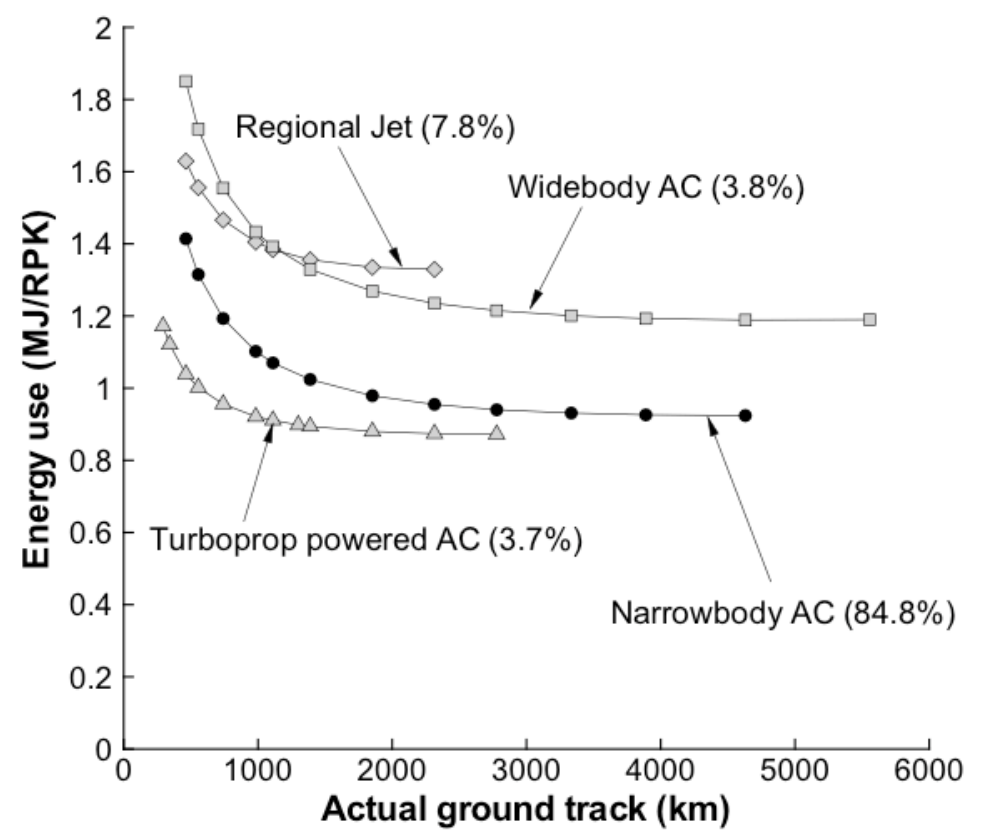

Figure 2 Dependence of energy use on flight range for representative aircraft (Vera Morales et al., 2011)

\subsection{Oxides of nitrogen}

Oxides of nitrogen (NOx) are associated with a range of air-quality-related human health impacts through several different paths. They also play a significant part in atmospheric chemistry, and thereby can enhance or mitigate the radiative heating of the earth, depending on altitude. The original IPCC report on aviation estimated their net contribution to global warming as positive and significant, albeit subject to considerable uncertainty (Prather and Sausen, 1999). However, Vera Morales et al. (2011) argue that this overstates their importance, and hence that local air quality is the only relevant consideration.

NOx is produced due to oxidation of nitrogen at the high temperatures in the engine combustor. Only a tiny proportion of the nitrogen available in the air entering the engine is converted, meaning that (unlike $\mathrm{CO}_{2}$ emissions) the amount of NOx generated depends not only on the mass of fuel burnt, but also on details of the engine combustor design. Broadly speaking, the higher the temperature and pressure at which combustion takes place, the greater the production of NOx.

The regulatory standards for aircraft-engine NOx emissions are set by the ICAO's Committee on Aviation Environmental Protection (CAEP). The current reference is known as CAEP/6, which represents a reduction of approximately $40 \%$ on the original limits set in the 1980s. (The exact figure depends on the engine pressure ratio.) Most modern engines meet this standard (ICAO, 2012), although there are wide variations even within a single model range. For example, the various versions of the CFM56-5B, which is used on the Airbus A320 family, have NOx characteristics ranging from $61 \%$ to $114 \%$ of the CAEP/6 limit. The ACARE Vision 2020 target of an $80 \%$ reduction relative to year 2000 would thus correspond to anywhere between $12 \%$ and $23 \%$ of the CAEP/6 limit if this engine were used as the reference. The least stringent value is roughly consistent with the NASA N+2 target (emissions below $25 \%$ of CAEP/6) for 2025 . Both comfortably surpass the reduction of the regulatory limit over the last thirty years.

\subsection{Noise}

Noise around airports is now well established as a major concern (He et al., Wolfe et al., this issue). It arises from a number of sources, of which those connected with the engines - the exhaust jet and the moving turbomachinery blades - have traditionally been dominant. Great progress, however, has been made since the first turbojets; Fig. 3 shows that the thrust-corrected noise has dropped by around $20 \mathrm{EPNdB}$ (corresponding to a hundred-fold reduction in sound power, and a four-fold reduction in perceived level). As a result, other sources have gained in significance, in particular when the engine is throttled back on approach to landing. These 'airframe noise' contributions arise because of unsteady airflows, mainly around the landing gear and the wing high-lift devices. 


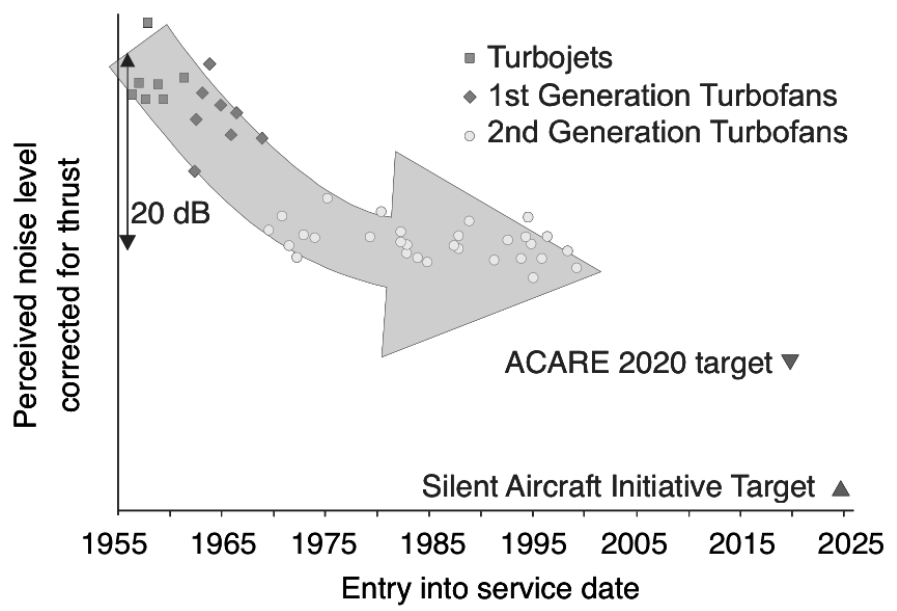

Figure 3 Historical evolution of aircraft noise levels (Hall, 2009)

The increase in source number has important implications for future noise-reduction prospects; the battle must now be fought on more fronts, and (due to the logarithmic nature of human noise perception) major gains are needed on all to achieve a significant overall advance. There is also an increasing likelihood of conflict with other design goals. The historical improvements shown in Fig. 3 are essentially associated with jet-noise reduction via increased engine 'by-pass ratio' (BPR), which has also led to significant fuel-efficiency improvements. High-BPR engines, however, have large nacelles, which increase the weight of, and drag on, the aircraft. Thus, while greater BPRs will always be beneficial for jet noise, their efficiency benefits will, at some point, be outweighed by the nacelle penalties. Debate over an 'optimum' BPR is ongoing; in fact, the influence of other parameters means that a single value probably cannot reliably be specified. (For example, the trade-off will differ between above-wing and below-wing mounting configurations, and BPR can also be altered by changing engine core size.) Similar issues arise for airframe sources; for example, the noise from the wing leading-edge high-lift devices ('slats') can be substantially reduced if they are replaced by a flap-like mechanism with no gap (a 'drooped leading edge'). The drooped leading edge is, however, less aerodynamically effective, so a larger wing is required, and fuel-burn increases. On the recently introduced Airbus A380, which has a drooped leading edge on the inboard wing, this penalty was accepted in order to meet noise targets.

Conflict between source-reduction measures is also possible, as shown by the following two examples. Increasing BPR to reduce jet noise requires a large fan, and the blade noise from this component is now a very significant part of the overall engine signature. Without technological developments here, there is a risk that raising BPR further could even be counter-productive from an acoustic perspective. Airframe noise sources can be attenuated very effectively by reducing landing speed. However, this would require a larger, and hence heavier, wing. The thrust needed to propel the aircraft would increase commensurately, and the engine noise would thus also rise.

The ACARE target of a 50\% reduction in year-2000 perceived noise by 2020 corresponds to a drop in level of 10 EPNdB. Given the clear evidence of diminishing returns in Fig. 3, and the added complication of airframe contributions, this goal is ambitious. To compare it with the corresponding NASA target, 42 EPNdB cumulative below Stage 4, we require some further background. Stage 3 noise regulations consist of (weight-dependent) limits on three noise measurements. Two are made during takeoff: 'takeoff' (microphone beyond runway) and 'sideline' (microphone to the side of runway). The third, 'approach', is measured on landing by a microphone ahead of the runway. Stage 4 uses the same values, but stipulates that the sum of the measurements (the 'cumulative' level) should be at least $10 \mathrm{EPNdB}$ below the sum of the limits. Figures for most aircraft in operation today are available at a database provided by France's Direction Générale de l'Aviation Civile (DGAC, 2012). The values depend not only on the aircraft model, but also on its specific variant and its engine type, so it is not possible to give a single, definitive, value for year-2000 noise. However, for the most recent major entrant at that point (the Boeing 777), the results are in the region of 20 EPNdB cumulative below Stage 3 , i.e. 10 EPNdB below Stage 4. The NASA N+2 target thus corresponds roughly to a further reduction of 32 EPNdB, which is in close agreement with the cumulative gain that would arise from ACARE's 10 EPNdB goal.

Further out, NASA's N+3 target would be achieved if a further 10 EPNdB were taken off each measurement, corresponding to a perceived noise level at roughly $25 \%$ of our nominal year-2000 baseline. This is more stringent than the FlightPath 2050 goal (35\%), and is envisaged much sooner (recall that the quoted N+3 horizon is 2030-35). Conversely, applying the same methodology to work backwards from the FlightPath $205035 \%$ perceived level, we can say that it is roughly equivalent to 55 EPNdB below Stage 4. 


\section{Future aircraft concepts}

As we have seen, aircraft fuel-burn, NOx generation and noise have reduced significantly since the onset of large-scale commercial aviation. Furthermore, in the cases of fuel-burn and noise at least, historical evidence of diminishing returns suggests that most of the straightforward possible improvements have already been made. Nonetheless, ACARE and NASA have put forward targets that, in broad terms, require advances of comparable magnitude again, on all three fronts, within forty years at most. It is therefore clear that these goals will not be achieved without a marked departure from a 'business as usual' approach. Recognition of this fact has motivated a number of future-aircraft concept studies over the past decade. Here they are described, in chronological order.

\subsection{Greener by Design}

The Royal Aeronautical Society's Greener by Design (GBD) initiative considered a wide range of possible future aircraft (Green, 2002). The analysis was based on the range equation, (1), combined with an allowance for take-off fuel, and used estimates for weight, lift-to-drag ratio and engine efficiency obtained from an extensive survey of the open literature. Also included was a consideration of hydrogen as an alternative, zero$\mathrm{CO}_{2}$, fuel. The use of hydrogen as a transportation fuel is challenging, because it is much less convenient to store than liquid hydrocarbons. In aircraft, it is additionally problematic due to its high volume (for a given energy content); as a result, the study suggested that the aircraft energy efficiency would suffer. Given that present-day hydrogen production is associated with significant emissions of $\mathrm{CO}_{2}$ (from steam-reforming of natural gas), this efficiency degradation would be problematic. It thus seems likely that the hydrogen-fuelled aircraft will only become a realistic possibility if zero- $\mathrm{CO}_{2}$ electricity becomes freely available, and for this reason it is not included in our subsequent comparisons.

The kerosene-fuelled configurations considered by the study consist of the standard 'tube-and-wing' (TW), the blended wing-body (BWB) and the laminar flying wing (LFW). The latter disposes with a separate fuselage in favour of a larger, wing-like, shape that combines payload transport with lift generation. The 'laminar' adjective refers to control of the flow next to the wing surface via suction, a technique which has the potential to yield very high reductions in the skin-friction component of drag. The BWB is an intermediate configuration, in which there is still an identifiable central body, but one that differs considerably from a conventional fuselage and is capable of contributing significant lift.

Several variants of the standard configuration were proposed. Most fundamentally, long-range $(15,000 \mathrm{~km}$ or $8,100 \mathrm{nmi})$ and medium-range $(5,000 \mathrm{~km}$ or $2,700 \mathrm{nmi})$ designs were investigated separately. For the mediumrange designs, an additional option was 'hybrid laminar flow control' (HLFC). This is essentially the same dragreduction technique as applied on the LFW, but with deliberate shaping of the aircraft surface to minimise suction area requirements. Its application to the fuselage is not considered practical; in this study, it was assumed on the tail surfaces, the engine nacelles, and possibly on the main wing.

For propulsion, both the standard, turbofan, engine and the 'open rotor' (or 'unducted fan') were suggested as possibilities. The latter consists of two counter-rotating, advanced-design propellors, as shown in Fig. 4. It is more fuel-efficient than a turbofan, essentially because it has a very high effective BPR without any nacelle drag. However, the jet-noise benefits of the BPR gain are offset by the removal of the nacelle, which increases the sound radiated by interactions between the blades and the airflow. Thus, while the design has been successfully demonstrated, it has not yet been manufactured commercially.

The study also proposed a novel alteration to the turbofan engine type, via introduction of the 'intercooled recuperative' (ICR) cycle. This would improve the fundamental thermodynamic efficiency of the design, by cooling its airflow between compression stages. However, the heat exchangers required would add significantly to the weight of the engine, thereby compromising the overall benefits.

Finally, expected advances in technology over time - chiefly improved engine thermal efficiency and reduced structural weight - were incorporated by specifying two parameter sets, one for 'present-day' (2001) and one for 2050. In our subsequent analysis, we will employ the 2050 results, using the 2001 values only for comparison. We will also limit the presentation of results for ICR engines to the standard configuration only, in part because the gains are small, and in part to allow closer comparison with the other studies reviewed here. A full list of the GBD configurations considered is given in Table A.1, in the appendix. 


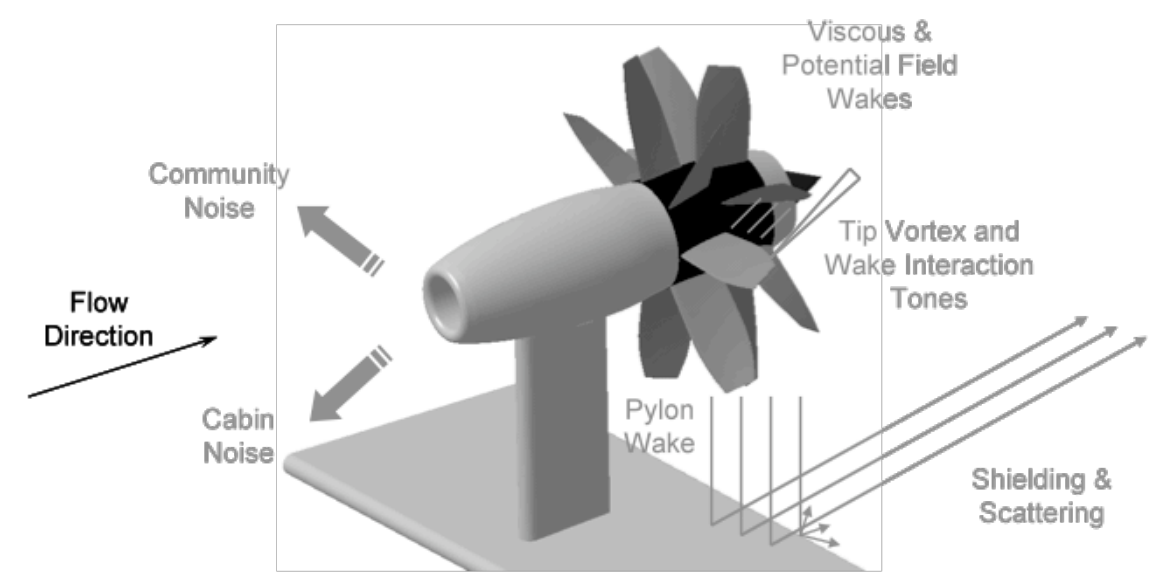

Figure 4 The open rotor engine showing the key noise issues for the configuration

\subsection{The Silent Aircraft Initiative (SAI)}

In this study (Dowling, 2007), noise was taken to be the primary driver of the design, with secondary requirements that fuel efficiency should be no worse than for current aircraft, and that the technology demands should permit entry into service at 2025 . The configuration chosen was a BWB, because it is inherently favourable for noise reduction. Specifically: it provides space to embed the engines in the airframe, which permits the use of high BPRs without nacelle penalties, and long, sound-absorbing inlet ducts; its large planform area shields ground observers from remaining engine noise (as long as the inlets are on the upper surface); it has better low-speed lift capability than a TW, eliminating some airframe noise sources and allowing for landing procedures that mitigate the others. It should also be noted that there is a fuel-efficiency benefit in taking the engine airflow from the slow-moving region near the airframe surface, albeit one that requires the turbomachinery to be more tolerant of flow 'distortion'. The final design is shown in Fig. 5.

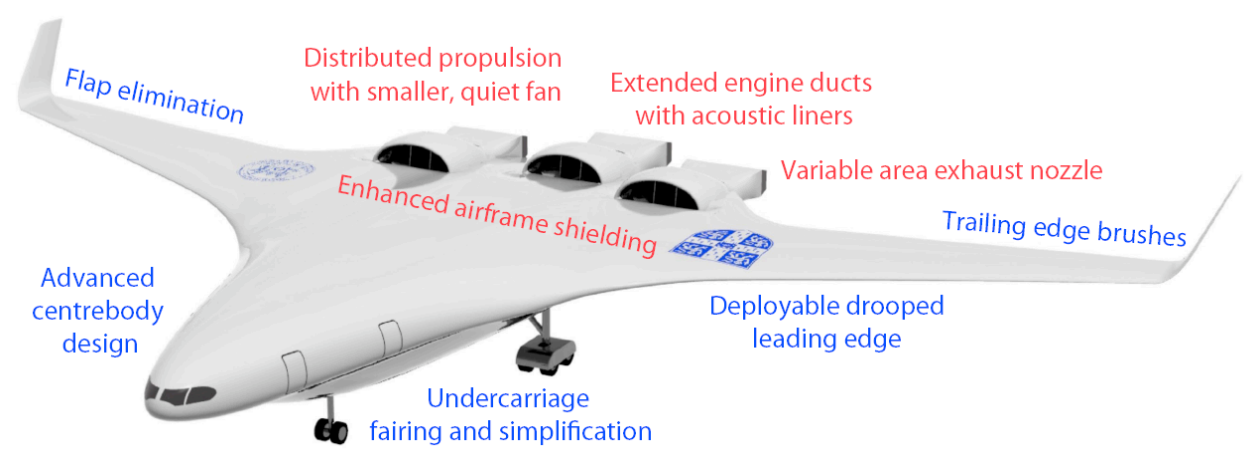

Figure 5 The Silent Aircraft design, SAX-40, with key noise-reduction technologies (Hall et al., 2009)

The embedded propulsion concept is technologically demanding, in that it is sensitive to inefficiencies in the duct flow, and requires the engine to cope with a distorted incoming air stream. For this reason, the SAI team additionally produced a 'low-risk' design, with pylon-mounted engines. This configuration will also be included for comparison purposes.

\section{$3.3 \quad$ NACRE}

NACRE (New Aircraft Concepts REsearch) was an integrated European project whose consortium consisted of the major airframe and engine manufacturers, as well as a number of research institutions and universities (Frota et al., 2011). Its aim was to develop understanding and analysis of advanced technologies in order to improve future concept modelling, but some specific configurations were considered in order to provide a context for the work. Of these, the 'Payload-Driven Aircraft' (a BWB) and the two 'Pro-Green' vehicles are relevant to this review. The Pro-Green designs are both TW, but with tail-mounted propulsion for noise shielding. On the first, PG1, the engines are contra-rotating turbofans; on the second (PG2) they are open rotors. In their most advanced incarnations they have 'forward-swept' wings, a layout which is structurally demanding, but which opens the possibility of 'natural laminar flow' (NLF); this is drag reduction via the same mechanism as HLFC, achieved without surface suction. Apart from the PG1 contra-rotating fan, which has a blade-noise benefit, other acoustic 
design features are the replacement of slats (by either leading-edge droop or a Krueger flap), and the use of a 'low-noise' undercarriage. It should, however, be noted that the hoped-for shielding benefit was not realised to any significant extent; a cumulative reduction of $4 \mathrm{EPNdB}$ across all three measurement locations is quoted. The nominal service-entry date for these concepts, and a reference baseline that was also specified, is 2015.

\subsection{NASA N+3 Studies}

Four major studies into $\mathrm{N}+3$ concepts with 2030-35 entry into service were funded by NASA; they were carried out by teams led by Boeing, GE/Cessna, Massachusetts Institute of Technology (MIT) and Northrop Grumman. Of these, GE and Cessna developed a small-aircraft concept that is outside the scope of this review. An additional, reduced-scale, $\mathrm{N}+3$ study, by the Conceptual Research Corporation (CRC), will, however, be included. Note that the quoted $\mathrm{N}+3$ targets have changed somewhat since these studies were carried out; at that time the goals for fuel-burn and NOx reduction were, respectively, $70 \%$ relative to a user-defined reference vehicle (now 60\% relative to year-2005 best-in-class) and 'in excess of 75\%' relative to CAEP 6 (now $80 \%$ ).

\subsubsection{Boeing SUGAR concepts}

Boeing (Bradley and Droney, 2011 ${ }^{3}$ ) chose to consider medium-size aircraft (passenger capacity 154, comparable to the present-day Airbus A320 and Boeing 737). Two baseline TW vehicles were defined; SUGAR Free (present-day technology) and Refined SUGAR (2030 technology). These were compared against three novel configurations; SUGAR High (a braced-wing design intended to achieve the greatest possible lift-to-drag ratio via induced-drag reduction), SUGAR Volt (a hybrid-electric version of SUGAR High), and SUGAR Ray (a BWB). All the 2030 aircraft were assumed to have drag-reducing technology (either NLF or HLFC as applicable, along with 'riblet' surfaces on the fuselage), advanced lightweight structures, and ultra-high-BPR turbofan engines. Their design range is $6,500 \mathrm{~km}(3,500 \mathrm{nmi})$, at a cruise Mach number of 0.7 , considerably below the values typical of current civil transports $(0.8-0.85)$. This speed reduction allows the use of an unswept wing, with greater span (for the same weight) than conventional designs. The benefits are twofold: the span increase improves the aircraft's lift-to-drag ratio (cf. Section 2.1), and the removal of sweep makes NLF a realistic prospect. The penalties are economic, due to increased travel time. However, according to Boeing's 'Current Market Outlook', a 6,500 km-range aircraft remains economically viable at Mach 0.7. On the acoustics front, in addition to increased engine BPR, advanced duct liners are employed, the slat is replaced by a Krueger flap, the landing gear is faired, and (unspecified) treatments are applied to the control surfaces and (presumably) trailing-edge flaps.

\subsubsection{The Conceptual Research Corporation study}

This work (Raymer et al., 2011) was based on the conceptual design techniques set out by Raymer (2006), and addressed fuel-burn only. The configuration proposed is a medium-size, tailless TW with rear-mounted openrotor engines. In the absence of a tail, the aircraft is inherently unstable, so the concept presupposes civilaviation acceptance of the artificially stabilising controllers that have become ubiquitous in fighter aircraft. It also proposes the use of a second technology that has reached military demonstrator level: the active aeroelastic wing. Here the wing is deliberately constructed with greater flexibility than is conventional, and its shape is altered according to the flight condition by an automatic control system. This, along with advanced structural design and manufacturing techniques, is expected to provide significant weight reductions. Finally, laminar flow over $25 \%$ of the surface is assumed, using either NLF, or HLFC if necessary.

\subsubsection{The MIT concepts}

Two designs, denoted D8.5 and H3.2, were proposed by MIT (Greitzer et al., 2011 ${ }^{4}$ ). The former, shown in Fig. 6 , is a medium-size aircraft carrying 180 passengers over a $5,600 \mathrm{~km}(3,000 \mathrm{nmi})$ range. Its key feature is a 'double-bubble' fuselage with an aerodynamically shaped outer skin. The lift generated by this component allows both wing and horizontal-tail areas to be reduced, yielding weight and aerodynamic efficiency benefits. It also provides a well-shielded mounting location for the ultra-high-BPR engines, whose close integration minimises nacelle penalties. Like the Boeing designs, advanced structural technologies are incorporated, and the cruising speed is reduced (to Mach 0.74 ) in order to take advantage of the benefits of an unswept wing. In this case, these include sufficient lift-generating capability to eliminate the need for a leading-edge high-lift device. The other airframe noise treatment is landing-gear fairing; the trailing-edge flaps are conventional.

The second MIT design, H3.2, is a long-range BWB carrying 354 passengers over 14,100 km (7,600 nmi) at a (conventional) cruise Mach number of 0.83. An ultra-high-BPR distributed propulsion system is embedded in

\footnotetext{
${ }^{3}$ See also http://aviationweek.typepad.com/files/boeing_sugar_phase_i_final_review_v5.pdf for an associated presentation.

${ }^{4}$ See also http://aviationweek.typepad.com/files/mit_n3 final_presentation.pdf for an associated presentation.
} 
the body, and advanced structural technologies provide weight savings. Aerodynamic efficiency improvements arise solely from the BWB configuration; significant regions of laminar flow are not expected.

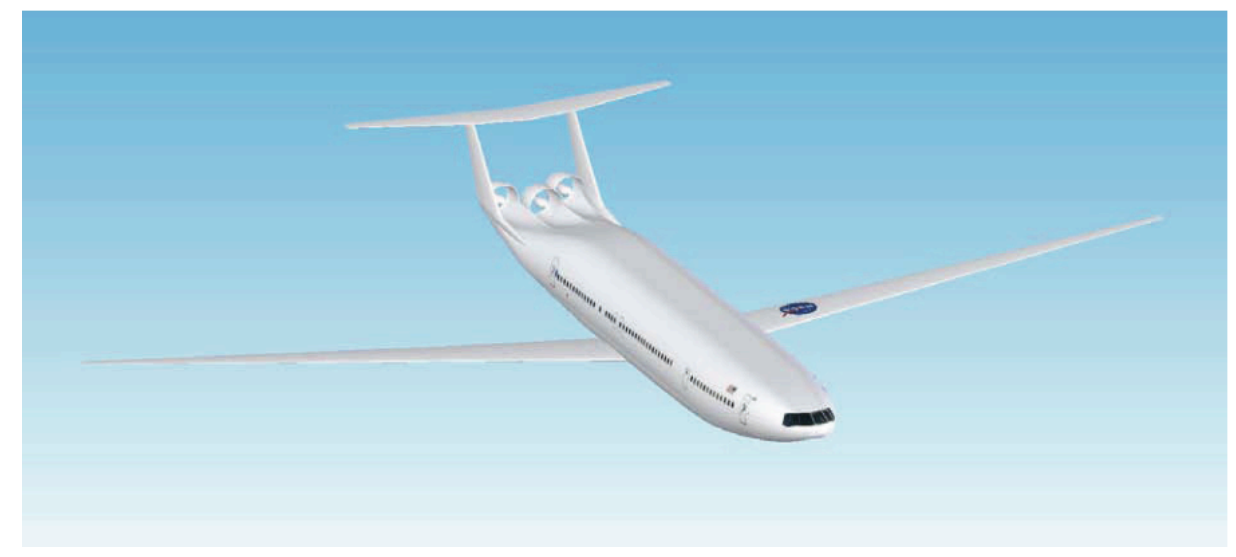

Figure 6 The MIT N+3 'Double Bubble' aircraft (Greitzer et al., 2011)

\subsubsection{The Northrop Grumman 'Preferred Vehicle'}

Northrop Grumman recommended a single conceptual design, denoted 'SELECT' (Bruner et al., 2010 $)$. It is conventional in its configuration, but aggressive in its incorporation of novel technologies. In particular: the engines are ultra-high-BPR, make use of the ICR cycle, and assume the availability of highly advanced materials (e.g. shape-memory alloys); the wing, although swept back, benefits from drag reduction via NLF on a significant proportion of its area and from active aeroelastic control; structural efficiency is improved via advanced composite technology. Noise is addressed via increased BPR and advanced liners for the engine, slat elimination and landing-gear fairings. Compared to the other $\mathrm{N}+3$ concepts, the design range and passenger count $-3,000 \mathrm{~km}(1,600 \mathrm{nmi})$ and 120 respectively - are both substantially lower, on the basis that future 'metroplex' operations will lead to a requirement for shorter, lower-capacity flights. The cruise Mach number is comparable to the MIT D8.5, at 0.75 .

\section{$3.5 \quad$ TOSCA}

The TOSCA project investigated the potential of technology to mitigate the climate impact of all major transport modes in Europe, in the period up to 2050. Noise was thus not part of the remit; the authors of the aircraft report (Vera Morales et al., 2011) also argued that NOx emissions were of little relevance in comparison to $\mathrm{CO}_{2}$, and therefore concentrated on fuel-burn.

The long lifespan of commercial civil aircraft means that a new type takes many years to achieve full fleet penetration. For this reason, the study set a service-entry date of 2025. It considered airliners of a size and range appropriate to intra-European transport: a narrow-body jet and a short-range turboprop, typified by two reference vehicles, the Airbus A320 and the Alenia ATR72.

A conservative view of the prospects for novel configurations in these fleet segments was taken; all the developments proposed are TW types. The first is an 'evolutionary' narrow-body, representing a 'business-asusual' improvement on the A320. The other two are open-rotor-powered aircraft, cruising either at the same Mach number (0.77) as the A320 — the 'fast open rotor' — or at Mach 0.66 - the 'reduced-speed open rotor'. Their fuel consumption figures come from a range-equation analysis (cf. Section 2.1). Estimates for the 2025 values of the parameters in Eq. (1) were obtained by surveying the views of professional experts, subject to scaling back by a $2 / 3$ factor in order to account for the empirical observation that the benefits anticipated for components in isolation are inevitably not achievable in combination (Schäfer et al., 2006).

For the turboprop type, the study noted that the thermodynamic efficiency of current engines is well below the levels attainable even today. The 2025 development - the 'evolutionary turboprop' - is thus simply a reengined version of the ATR72, with unchanged aerodynamic and structural technology.

\subsection{NASA N+2 Studies}

The most recent investigations reviewed here were commissioned by NASA's 'Environmentally Responsible Aviation' project. They are for vehicles at the $\mathrm{N}+2$ stage, with entry into service envisioned at 2025 . Three

\footnotetext{
${ }^{5}$ See also http://aviationweek.typepad.com/files/northrop_grumman_final.pdf for an associated presentation.
} 
teams, led by Boeing, Lockheed Martin and Northrop Grumman, took part. Each was required to evaluate three configurations: a 1998 baseline sized on the Boeing 767 (224 passengers, 14,800 km (8,000 nmi) range, Mach 0.85 cruise), and two future designs for the same mission. One of the latter was restricted to the conventional, TW, layout, but for the other — the 'Preferred System Concept' - complete freedom was allowed.

\subsubsection{Boeing team concepts (Bonet, 2012)}

Boeing anticipated the use of the following technologies on their 2025 TW: HLFC, riblets and increased wing aspect ratio for drag reduction; either advanced turbofan (Rolls Royce) or geared turbofan (Pratt and Whitney) propulsion; complete replacement of aluminium by composite materials in the structure. Noise reduction is provided via unspecified acoustic treatments on the wing leading edge, the landing gear and the engines, and by the use of a Krueger flap instead of a conventional leading-edge slat.

For their Preferred System Concept, the team specified a BWB. The advanced technologies assumed are very similar to those on the TW, except that an open-rotor engine replaces the advanced turbofan option, and noise performance is enhanced by the inherent shielding capabilities of the BWB planform. In addition, the conventional, slotted, trailing-edge flap is replaced by a plain version, presumably for noise reasons.

\subsubsection{Lockheed Martin team concepts (Martin, 2012)}

Lockheed Martin's TW features laminar flow control (whether NLF or HLFC is not specified), an advanced turbofan engine and a fully composite structure. Airframe-noise reduction treatments consist of 'continuous moldline' trailing-edge flaps, a filled-gap leading-edge slat, landing-gear fairings and shape-memory-alloy serration on the chevrons at the rear of the nacelle. Engine noise is lowered via increased BPR (doubled from the Rolls Royce Trent 800 baseline of 6).

The team's Preferred System Concept is a box-wing configuration, on which a second pair of wings links the tips of the conventional wings to the vertical tail surface (Fig. 7). The technology assumed is the same as for the TW, with the exception of the engines. These are mounted on the higher, rear, wings, allowing space for an 'Ultrafan' engine with BPR five times that of the Trent 800. Nacelle size is thus a concern; even with laminar flow control specified to reduce its drag, the team note that further optimisation is necessary, and that the best compromise may be at a lower BPR.

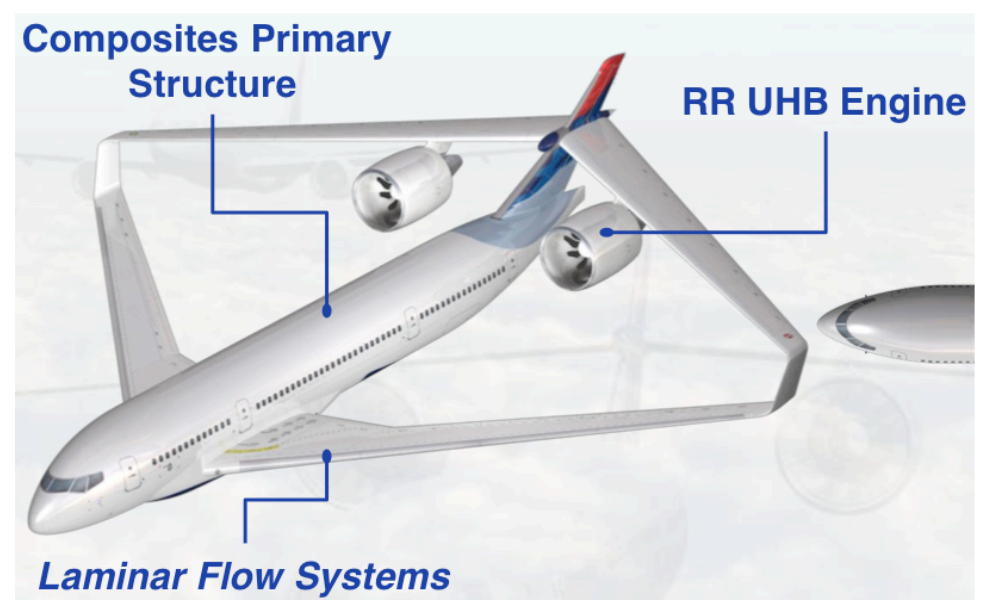

Figure 7 Lockheed Martin's box-wing concept for the N+2 study (Martin, 2012)

\subsubsection{Northrop Grumman team concepts (Drake, 2012)}

The most significant novel technologies on Northrop Grumman's TW are laminar flow control of some form, a basket of (unspecified) advanced propulsion technologies, a composite wing and an 'advanced' fuselage structure. No innovations specifically targeted at noise are cited, although it is likely that some of the propulsion technologies will be beneficial. The same refinements appear on the team's Preferred System Concept, a flying wing, but the engines are now embedded, allowing a higher BPR without nacelle drag/weight penalties. Apart from fuel efficiency, this will also be beneficial for noise, as will the flying-wing planform (cf. Section 3.2). 


\subsection{Summary}

A wide range of concepts and novel technologies is covered by the studies described here. In the appendix, summary tables are provided in order to make comparison easier. Table A.1 lists the concepts selected for analysis in this paper, and the identifying labels assigned to them. The respective missions are set out in Table A.2, while Tables A.3 and A.4 provide synopses of the technologies assumed for improving fuel-burn and noise performance respectively. No corresponding list is given for NOx reduction, because most of the studies that address this issue give little specific detail on the advanced combustor designs that they assume.

\section{Comparisons of emissions estimates}

\subsection{Carbon dioxide}

The absence of regulatory standards for this category means that there is no single established metric for aircraft fuel consumption. Furthermore, even when direct comparison between different studies is possible, there may be considerable disagreement in absolute numerical values. (For example, both the CRC and MIT N+3 studies use the Boeing 737-800 as a reference, and there is sufficient information in the former to derive the payload fuel efficiency for comparison with the value given by the latter. The respective figures $-9.12 \mathrm{~kJ} / \mathrm{kgkm}$ and 7.43 $\mathrm{kJ} / \mathrm{kgkm}$ - differ by $19 \%$.) For this reason, we shall confine our analysis to relative benefits; i.e. any given future configuration will be characterised by its performance relative to a baseline obtained in the same study. Two categories of baseline will be employed: either a present-day TW, or a 'contemporary' TW (i.e. one entering service at the same date as the proposed future configuration). Comparisons in the first category will be further split into those for future TW aircraft, and those for novel configurations.

\subsubsection{Future TW aircraft}

The majority of the concepts in this category are for service-entry dates of either 2025 or 2050 . The former group consist of the TOSCA and the NASA ERA $\mathrm{N}+2$ vehicles. The $\mathrm{N}+2$ specification required consideration of a 14,800 km (8,000 nmi), 224-passenger, twin-aisle TW (typified by the Boeing 767) as a present-day baseline. The TOSCA project modelled the Airbus A320 and Alenia ATR72 as references for, respectively, the narrow-body and turboprop classes. The results are shown in Fig. 8, arranged according to an informal assessment of technological novelty. Thus the TOSCA concepts are furthest left, with the most demanding being the higher-speed open rotor. All the N+2 aircraft are deemed more complex, due to their use of laminar flow control for drag reduction. Substantial improvements, of 38-50\%, are predicted for these vehicles. Similar gains are forecast by TOSCA for the reduced-speed open rotor and the re-engined turboprop. (Note that, in absolute terms, the latter is slightly better than the former, as the baseline turboprop is more efficient than the baseline narrow-body.) Nonetheless, only one of the concepts shown is expected to meet the Vision $2020 / \mathrm{N}+2$ $50 \%$ goal.

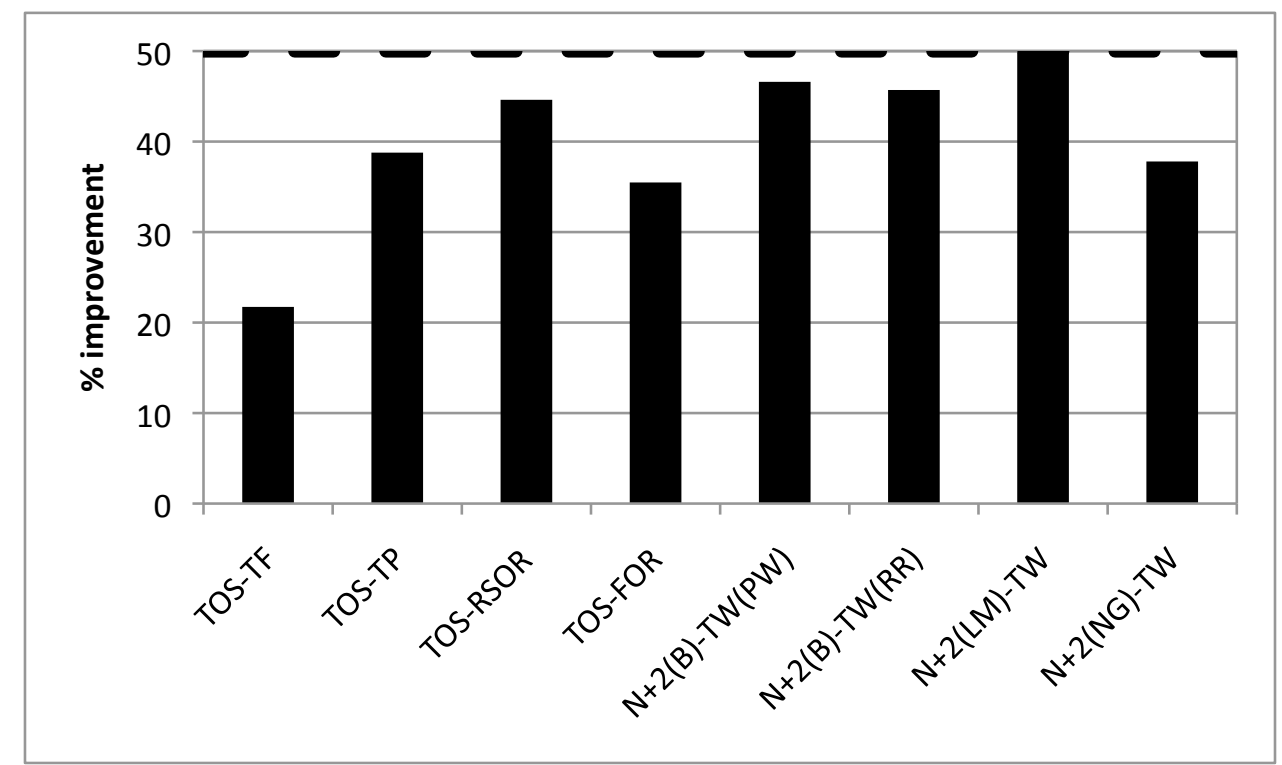

Figure 8 Percentage reductions in fuel consumption for 2025 service-entry TW concepts. TOSCA study: TF, turbofan; TP, turboprop; RSOR, reduced-speed open rotor; FOR, fast open rotor. NASA ERA N+2 studies: (B), Boeing; (LM), Lockheed Martin; (NG), Northrop Grumman; all turbofans, but (B)TW(PW) is geared. Dashed line shows ACARE Vision 2020/NASA N+2 target. 
The conventional configurations with 2050 service-entry all come from the GBD study. Their predicted fuelburn reductions are shown in Fig. 9. In these cases, the references are the present-day TWs of the same range capability. Thus, although the long-range configurations - GBD-TWL and GBD-TWL(ICR) - show greater relative improvements, they remain less efficient in absolute terms than their medium-range counterparts. Of the novel technologies proposed, the ICR engine cycle has only a small influence (due to its associated weight penalty), while HLFC is more effective, especially in its more extensive form. Relative to the 2025 predictions, these results appear, at first sight, more conservative. However, the only direct comparison available is for a medium-range aircraft with 'business-as-usual' improvements: TOS-TF vs GBD-TWM, with 22\% and 28\% reductions respectively. The other turbofan-powered 2025 vehicles are the long-range $\mathrm{N}+2$ concepts, and these employ laminar flow. The long-range GBD vehicle would probably achieve comparable gains under the same assumption. Even so, it would not approach either the NASA N+3 or Flightpath 2050 targets $(60 \%$ and $75 \%$ respectively).

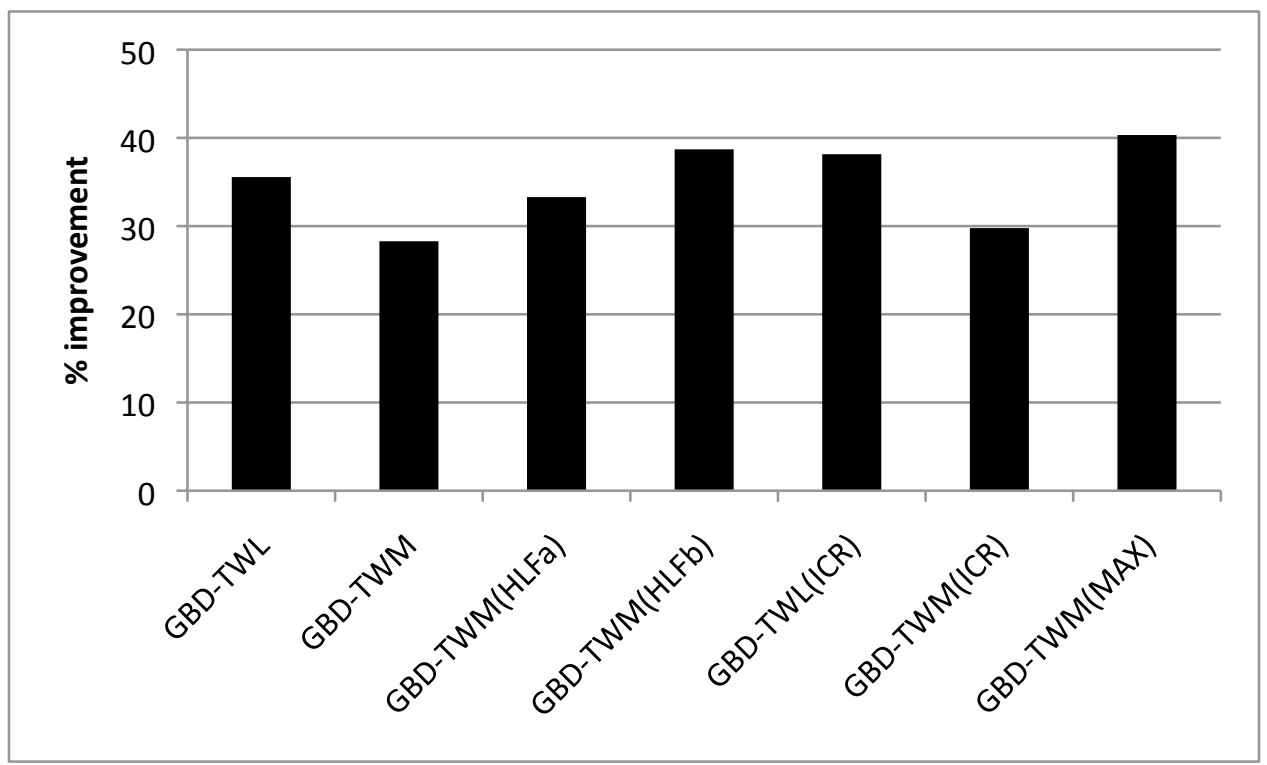

Figure 9 Percentage reductions in fuel consumption for GBD 2050 service-entry TW concepts. TWL and TWM are conventional long- and medium-range turbofans. Novel technologies: HLFa, HLFC on fin, tail and nacelle; HLFb, HLFa plus wing; ICR, ICR engine cycle; MAX, HLFb and ICR combined.

Two other TW-development predictions are available in the studies reviewed here, for the 2035 service-entry vehicles proposed in the Boeing and Northrop Grumman $\mathrm{N}+3$ studies. These have quoted fuel-burn reductions of $44 \%$ and $64 \%$ respectively. In terms of technological novelty, the Boeing concept is broadly comparable with the N+2 2025 TWs, while the Northrop Grumman proposal additionally invokes an ICR engine cycle and an aeroelastic wing.

\subsubsection{Novel aircraft relative to the present day}

Direct comparisons between novel configurations and present-day TW counterparts were made by the GBD, $\mathrm{N}+2, \mathrm{~N}+3$ and SAI studies. Their predictions are summarised in Fig. 10. All the aircraft are kerosene-burning, apart from the hybrid-electric N+3(B)-BW(HE), whose fuel consumption represents only a part of its energy use (the remainder being provided by electric batteries). Note, however, that its claimed performance is subject to considerable doubt in the light of detail inconsistencies in the study report. In particular, on the basis of cited engine operating parameters in cruise, it burns about $20 \%$ less fuel than its pure-turbofan twin, $\mathrm{N}+3(\mathrm{~B})-\mathrm{BW}$. In contrast, the report summary values shown in Fig. 10 imply $40 \%$ superiority, a huge discrepancy. The summary also gives a 20,000lb lower weight for the hybrid-electric version, despite stated additions of 9,500lb for the engine, $6000 \mathrm{lb}$ for battery mounting and wiring, and an unspecified amount for the batteries themselves. These are offset by an estimated 15,000lb potential reduction for the wing, but this would (at most) bring the aircraft back down to the same weight as its twin. In any case, the legitimacy of applying this reduction to only one of the pair must also be questioned. For these reasons, only the pure-turbofan version of this configuration will be considered subsequently.

Among the kerosene-burning concepts, the LFW, tailless TW and double-bubble have the greatest predicted benefits, in the region of the NASA N +3 target. The remainder have reductions lying in the range $37-54 \%$, with 
the exception of the SAI concepts, whose lower figures reflect the dominance of noise concerns in their design. Even discounting these, the remainder group contains a majority of BWB configurations, with their predicted improvements spanning the $37-54 \%$ range. Here the MIT N+3 concept stands out, in that (like the GBD BWB) it does not apparently rely on laminar flow, but is as efficient as the Boeing $\mathrm{N}+2$ design (which does). The GBD study also provides estimates of the benefit of open-rotor propulsion; as for the TOSCA TWs (cf. Fig. 8), a significant impact is expected. (Note, however, that Boeing anticipate much smaller gains relative to a geared turbofan alternative.) Among the remaining configurations, the Lockheed Martin N+2 box-wing concept has the best performance prediction, at 50\%, while the Boeing $\mathrm{N}+3$ braced-wing aircraft and the Northrop Grumman flying wing reductions are around $40 \%$. For the latter, the contrast with the LFW figures reflects the benefit of full, rather than partial, flow laminarisation. Finally, none of the designs is forecast to meet the FlightPath 2050 target.

\subsubsection{Novel aircraft relative to TW contemporaries}

The other relevant comparison for a novel aircraft concept is against a putative future conventional vehicle of the same generation. This information is unavailable for the CRC and MIT N+3 studies, and for the SAI designs. It is, however, provided for the NACRE configurations. The results are summarised in Fig. 11. For the vehicles already shown in Fig. 10 there is, in principle, no new information here, but the presentation is useful for assessing the inherent merit of a novel configuration. In particular, the LFW now appears markedly superior to the turbofan-powered BWB, which in one case is even predicted to perform worse than its TW contemporary. The most optimistic BWB estimate in this set comes from the NACRE project, and shows a relative benefit of $19 \%$. The other NACRE configurations accrue a relatively small (4\%, cf. PG1) improvement from the amount of laminar flow anticipated on the advanced wing, but a significant gain from the switch to open-rotor propulsion (cf. PG2).

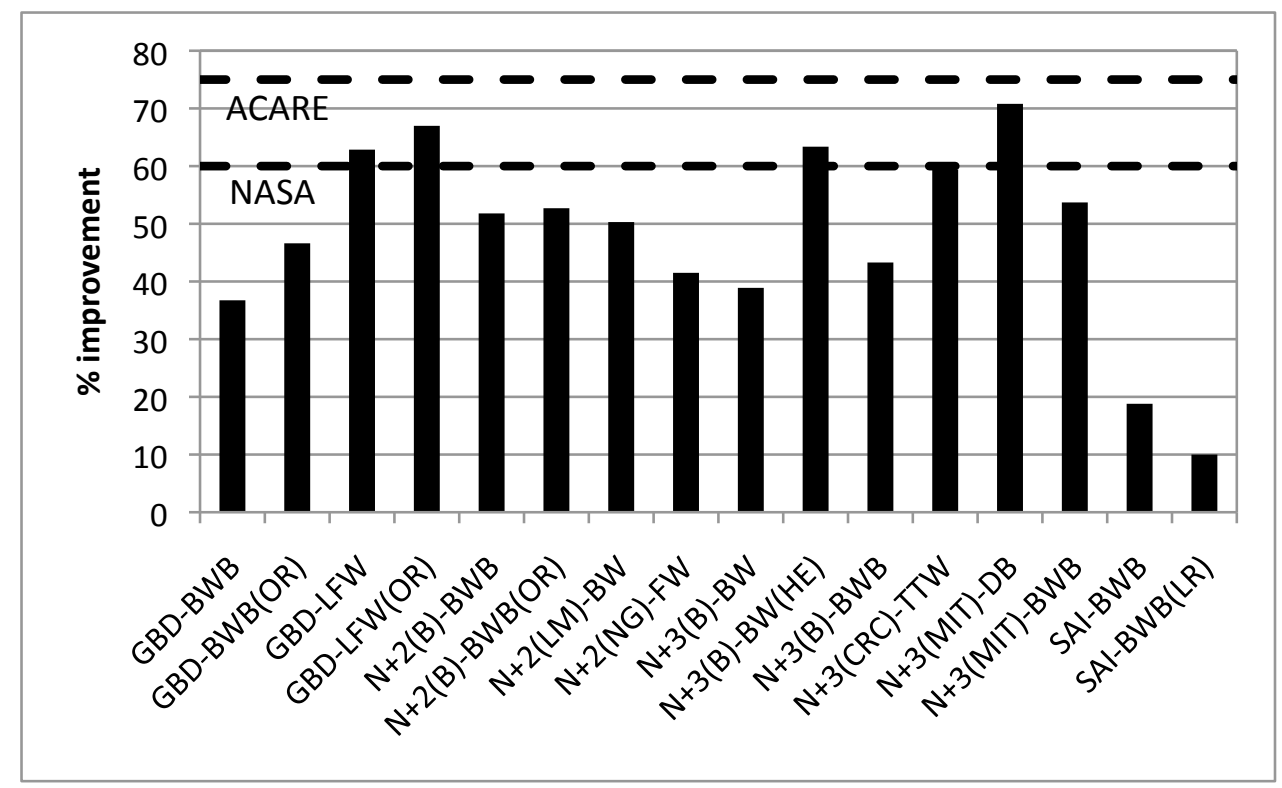

Figure 10 Percentage reductions in fuel consumption for novel-configuration concepts, relative to their presentday TW counterparts. GBD study: BWB and LFW with turbofans or open rotors (OR). NASA ERA N+2 studies: (B)-BWB, Boeing BWB with geared turbofans or open rotors (OR); (LM)-BW, Lockheed Martin box-wing with geared turbofans; (NG)-FW, Northrop Grumman flying wing with embedded turbofans. NASA N+3 studies: (B)-BW, Boeing braced-wing with pure or hybrid-electric (HE) turbofans; (B)-BWB, Boeing BWB with turbofans; (CRC)-TTW, tailless TW with open rotors; (MIT)-DB, double-bubble with integrated turbofans; (MIT)-BWB, BWB with embedded, distributed propulsion. Silent Aircraft Initiative: noise-optimised BWB with embedded or conventional (LR) turbofans. ACARE FlightPath 2050 and NASA N+3 targets are shown as dashed lines. 


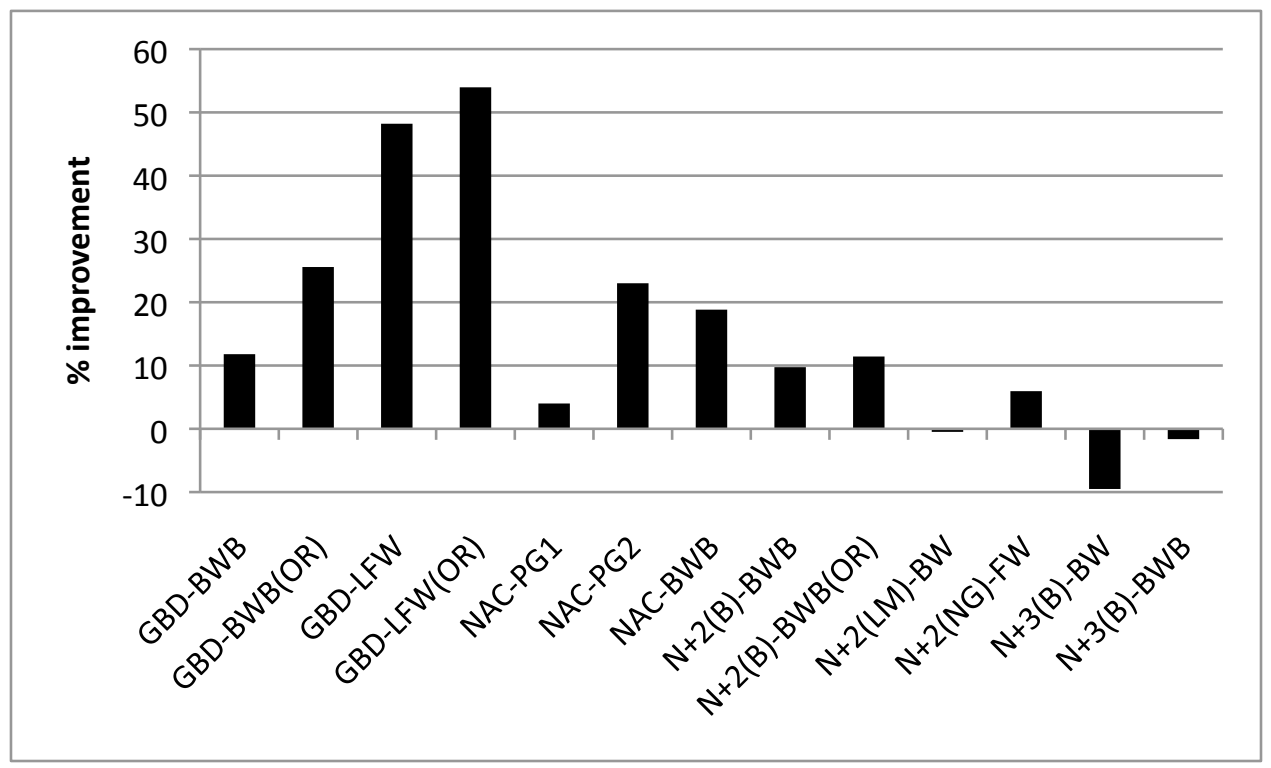

Figure 11 Percentage reductions in fuel consumption for novel-configuration concepts, relative to their contemporary TW counterparts. GBD study: BWB and LFW with turbofans or open rotors (OR). NACRE study: PG1, turbofan TW with forward sweep; PG2, open-rotor version of PG1; BWB, turbofan BWB. NASA ERA N+2 studies: (B)-BWB, Boeing BWB with geared turbofans or open rotors (OR); (LM)-BW, Lockheed Martin box-wing with geared turbofans; (NG)-FW, Northrop Grumman flying wing with embedded turbofans. NASA N+3 studies: (B)-BW, Boeing braced-wing with turbofans; (B)-BWB, Boeing BWB with turbofans.

\subsubsection{Analysis}

Further insight into the fuel-consumption results can be gained from the values of the fundamental parameters that appear in the range equation: the empty-to-payload weight ratio, the engine efficiency and the lift-to-drag ratio. These are broadly indicative of, respectively, the structural, propulsive and aerodynamic performance of the aircraft. (Note, however, that there is also a strong influence of design range on the weight ratio, because additional fuel displaces payload. Equally, this variable can improve if better engine or aerodynamic efficiency allows payload to be substituted for fuel.) Table 2 presents the available data. Both absolute values and their associated relative improvements are given where possible, but in some cases only figures for one or the other can be found. In all cases, the reference vehicles are the present-day TWs used to provide the baselines for Figs. $8-10$.

It is immediately clear that the absolute values of the weight ratio vary substantially. For the TW aircraft, this is largely attributable to the influence of mission range (compare, for example, the long-range $\mathrm{N}+2$ studies with the medium-range TOSCA narrow-body). The anticipated gains in this category arise entirely from the use of advanced composite materials instead of aluminium; despite this common feature, the predicted improvements vary widely, from $5 \%$ to $31 \%$. For the other configurations, large differences in absolute values are again evident, and are now less clearly associated with range. Among the BWBs, the longest-range version for which there is data - the $\mathrm{N}+3$ (MIT)-BWB - has one of the lowest weight ratios. This is partly because this configuration is most effective when large (it is very difficult to accommodate enough passengers to utilise its full payload capability when it is smaller), but also reflects the considerable uncertainty in weight estimation for its novel structure, especially the non-cylindrical, pressurised, passenger cabin. Similar comments apply for the flying wings. The box-wing and braced-wing estimates are likely to be more reliable; here the former $\mathrm{N}+2$ (LM)-BW - is comparable to its TW contemporary, whereas the structural demands of the latter $\mathrm{N}+3$ (B)-BW - lead to a much greater weight ratio than $\mathrm{N}+3(\mathrm{~B})-\mathrm{TW}$. Finally, the benefit of reducing or eliminating the rear tail is evident in the low values predicted for the CRC tailless TW and the MIT doublebubble. It should, however, be noted that the figure of 1.3 for the latter is remarkably small. Present-day aircraft have a payload-related weight (central fuselage, furnishings, etc) approximately equal to that of the payload itself (Green, 2002). For the double-bubble, this ratio appears to have dropped from 1 to 0.56, a huge reduction. 
Table 2 Range-equation parameters for the future aircraft concepts

\begin{tabular}{|c|c|c|c|c|c|c|}
\hline \multirow[t]{2}{*}{ Identifier } & \multicolumn{2}{|c|}{ Weight ratio $W_{e} / W_{p}$} & \multicolumn{2}{|c|}{ Engine efficiency } & \multicolumn{2}{|c|}{ Cruise L/D } \\
\hline & Value & $\%$ gain & Value & $\%$ gain & Value & $\%$ gain \\
\hline GBD-TWL & 2.1 & 31 & 0.40 & 8 & 20 & 7 \\
\hline GBD-TWM & 1.7 & 24 & 0.40 & 8 & 18 & 8 \\
\hline GBD-TWL(ICR) & 2.3 & 25 & 0.43 & 17 & 20 & 7 \\
\hline GBD-TWM(ICR) & 1.8 & 18 & 0.43 & 17 & 18 & 7 \\
\hline GBD-TWM(HLFa) & 1.7 & 24 & 0.40 & 8 & 20 & 17 \\
\hline GBD-TWM(HLFb) & 1.7 & 24 & 0.40 & 8 & 22 & 27 \\
\hline GBD-TWM(MAX) & 1.8 & 19 & 0.43 & 17 & 22 & 27 \\
\hline GBD-BWB & 1.5 & 32 & 0.40 & 8 & 19 & 14 \\
\hline GBD-BWB(OR) & 1.5 & 32 & 0.45 & 22 & 20 & 20 \\
\hline GBD-LFW & 1.8 & 19 & 0.40 & 8 & 37 & 117 \\
\hline GBD-LFW(OR) & 1.8 & 20 & 0.45 & 22 & 37 & 117 \\
\hline NAC-BWB & $2.4(4.2)^{1}$ & & & & 23 & \\
\hline $\mathrm{N}+2(\mathrm{~B})-\mathrm{TW}(\mathrm{PW})$ & & 14 & & 18 & & \\
\hline $\mathrm{N}+2(\mathrm{~B})-\mathrm{TW}(\mathrm{RR})$ & & 22 & & 15 & & \\
\hline$N+2(B)-B W B$ & & 21 & & 18 & & \\
\hline$N+2(B)-B W B(O R)$ & & 19 & & 20 & & \\
\hline$N+2(L M)-T W$ & 3.8 & 24 & & 17 & & 21 \\
\hline$N+2(L M)-B W$ & 3.8 & 23 & & 22 & & 16 \\
\hline$N+2(N G)-T W$ & 5.1 & 11 & & & & \\
\hline$N+2(N G)-F W$ & 4.5 & 21 & & & & \\
\hline$N+3(B)-T W$ & 2.5 & 19 & 0.32 & & 22 & 22 \\
\hline$N+3(B)-B W$ & 3.7 & -18 & 0.36 & & 26 & 44 \\
\hline$N+3(B)-B W B$ & 3.6 & -16 & 0.36 & & 27 & 52 \\
\hline $\mathrm{N}+3(\mathrm{CRC})-\mathrm{TTW}$ & 1.5 & 30 & 0.44 & & 25 & 52 \\
\hline $\mathrm{N}+3(\mathrm{MIT})-\mathrm{DB}$ & 1.3 & 42 & 0.49 & 40 & 25 & 67 \\
\hline $\mathrm{N}+3(\mathrm{MIT})-\mathrm{BWB}$ & $1.6(2.8)^{1}$ & & 0.41 & & 24 & \\
\hline$N+3(N G)-T W$ & 1.8 & 25 & 0.41 & & 21 & \\
\hline SAI-BWB & 4.0 & & 0.40 & & 25 & \\
\hline SAI-BWB(LR) & 4.2 & & 0.37 & & 25 & \\
\hline TOS-TF & 2.3 & 5 & 0.35 & 11 & 19 & 14 \\
\hline TOS-FOR & 2.3 & 5 & 0.41 & 31 & 20 & 18 \\
\hline TOS-RSOR & 2.3 & 5 & 0.42 & 33 & 22 & 35 \\
\hline TOS-TP & 1.8 & 0 & 0.41 & 61 & 16 & 0 \\
\hline
\end{tabular}

${ }^{1}$ The payload for these concepts has a significant cargo component. The weight ratio for passengers and luggage only is given in brackets.

The engine efficiencies show generally greater consistency than the weight ratios. Nonetheless, there is still considerable variation in the improvements expected in the turbofan type. This is probably due in part to differences in the predictions for future BPR, which range from no change (for the GBD vehicles) to a five-fold increase (for the $\mathrm{N}+2(\mathrm{LM})-\mathrm{BW}$ ). However, it is also a reflection of inherent uncertainty in the forecasting. In 
this context, the historical figures for engine efficiency presented by Lee et al. (2001) are of interest; they suggest that all but the least optimistic predictions (by GBD, for $8 \%$ improvement at 2050) would represent a departure from trend. There is general agreement that open-rotor engines will be more fuel-efficient than turbofans, but again some disagreement over the extent of their superiority. The highest absolute value is 0.49 , for the MIT double-bubble; were this for a standard installation, it would be tempting to dismiss it as an outlier, but it in fact reflects the benefit of ingesting the slow-moving air next to the fuselage (cf. Section 3.2). The highest relative gain is for TOSCA's re-engined turboprop, because of the poor current performance of the engines on this type.

Turning to lift-to-drag ratio, the studies are unanimous in predicting improvements over typical values today in the 15-18 range. According to GBD, these will arise solely from reductions in the skin-friction component, and will be below 10\% unless HLFC is employed, in which case they could give a 27\% increase to a value of 22 . The Boeing and Northrop Grumman N+3 TW studies concur with this figure, although they also invoke reductions in lift-dependent drag (via increased wing aspect ratio) to achieve it. At the other end of the spectrum, TOSCA's aircraft assume no change in the skin-friction contribution, with all the improvements coming from aspect-ratio increase. This yields a value of 19 for the turbofan-powered TW, but higher values for the open-rotor vehicles. This is in part because the latter have optimum cruise speed at the airframe's maximum lift-to-drag ratio (unlike turbofans, whose propulsion efficiency improves with speed), but also because the reduced-speed variant needs no wing sweep-back, and the resulting weight benefit can be used to increase aspect ratio further without a structural penalty.

The BWB configurations are generally estimated to have somewhat higher lift-to-drag ratio than the TW vehicles; this is, in fact, the key feature of the concept from a fuel-burn aspect. (In this light, the GBD values seem likely to be underestimates.) Note, however, that the CRC tailless TW and the MIT double-bubble are predicted to match the BWB in aerodynamic efficiency. Once again, reducing tail area has proved beneficial; in addition, both aircraft use unusually high aspect ratio wings to minimise the lift-dependent drag. The MIT vehicle, like the TOSCA reduced-speed open rotor, has no sweep, while the CRC design invokes an active aeroelastic wing to avoid the usual weight penalties associated with extreme aspect ratio.

Finally, the LFW configuration has a predicted lift-to-drag ratio that is spectacularly greater than any other, and that typically arouses scepticism among industry experts. Nonetheless, it is consistent with the assumption that full laminarisation of the surface flow is achieved on a planform that is inherently free of most of the excrescences found on a TW design. Whether this is attainable in practice remains an open question, which is presumably why the concept has not found favour in any of the recent industrial studies.

\subsection{Oxides of nitrogen}

Figure 12 shows the available predictions for LTO cycle NOx emissions relative to CAEP 6 . In considering these results, it must be borne in mind that present-day aircraft are typically already below CAEP 6; margins of $9 \%, 21 \%$ and $31-32 \%$ are given by, respectively, the Northrop Grumman $(\mathrm{N}+2)$, Boeing $(\mathrm{N}+3)$ and $\mathrm{MIT}(\mathrm{N}+3)$ studies (cf. also Section 2.2). Nonetheless, there is widespread agreement that further significant reductions, of the order of the NASA N+3 targets, are achievable. Little, if any, specific detail is provided in the various studies, but, where general descriptions are given, they are consistent: 'lean-burn' combustors will reduce combustion temperatures by increasing the local ratio of air to fuel. In their more advanced forms, they will split the fuel flow into different streams, which will be directly injected into the desired combustion locations. 


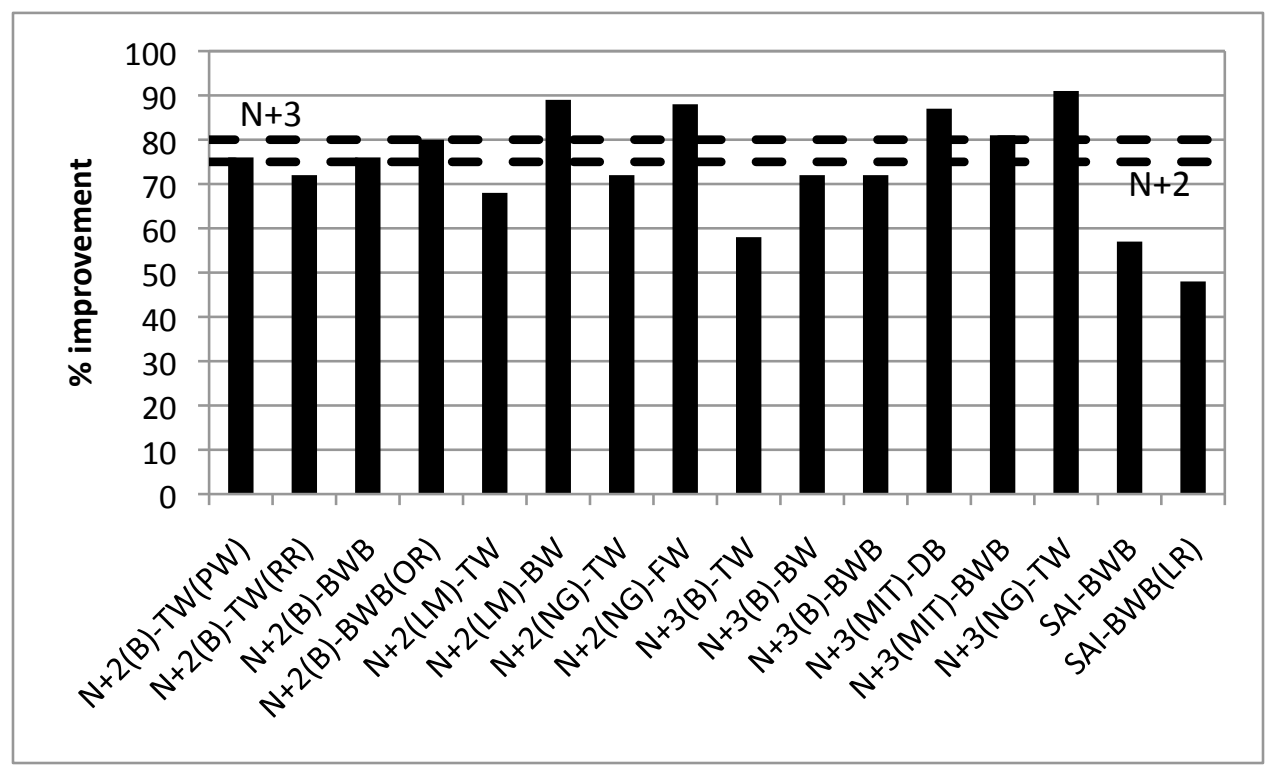

Figure 12 Percentage reductions in LTO cycle NOx emissions relative to CAEP 6 limits. NASA ERA N+2 studies: (B)-TW, Boeing TW with geared (PW) or conventional (RR) turbofans; (B)-BWB, Boeing BWB with geared turbofans or open rotors (OR); (LM)-TW, Lockheed Martin TW with turbofans; (LM)-BW, Lockheed Martin box-wing with geared turbofans; (NG)-TW, Northrop Grumman TW with turbofans; (NG)-FW, Northrop Grumman flying wing with embedded turbofans. NASA N+3 studies: (B)-TW, Boeing TW with turbofans; (B)-BW, Boeing braced-wing with turbofans; (B)-BWB, Boeing BWB with turbofans; (MIT)-DB, double-bubble with integrated turbofans; (MIT)-BWB, BWB with embedded, distributed propulsion; (NG)-TW, Northrop Grumman TW with turbofans. Silent Aircraft Initiative: noise-optimised BWB with embedded or conventional (LR) turbofans. Dashed lines show NASA N+2 and N+3 targets.

\subsection{Noise}

Figure 13 shows predicted noise reductions, in cumulative EPNdB relative to Chapter 4 limits. All the studies shown provided their results in this form, with the exception of NACRE, which gave single values relative to a notional 2015 narrow-body baseline. Cumulative reductions for the NACRE configurations were estimated by assuming that the individual values applied equally to all three Chapter 4 measurements; a representative baseline value was taken from the most recent A320/CFM56 combination at 6.5 EPNdB (DGAC, 2012).

First, it should be noted that the Northrop Grumman N+3 study's prediction of 70 EPNdB reduction is a clear outlier for the TW configuration. If it is discounted, the results imply that novel configurations, capable of providing a significant degree of shielding (and, preferably, engine embedding) are necessary to approach NASA's N+3 target. Such configurations are also more amenable to the substitution or elimination of high-lift devices, simplifying the problem of airframe noise reduction. For the remaining contributor under this heading, the undercarriage, there is general agreement that fairings will be necessary, whatever the configuration. Finally, as noted previously, all airframe noise sources can be reduced by flying slower on landing approach, and several studies invoke this strategy.

Two direct comparisons between turbofan and open-rotor propulsion are available, from the NACRE and Boeing $\mathrm{N}+2$ studies. Both concur that the latter will be noisier, although not to the extent that present-day turbofan levels are exceeded.

Boeing's N+3 study also gives noise figures, but relative to their reference 1998 vehicle rather than Chapter 4. Improvements of $16 \mathrm{~dB}, 22 \mathrm{~dB}$ and $37 \mathrm{~dB}$ are quoted for the future baseline, the braced-wing configuration and the BWB respectively. Although it is not explicitly stated, these are presumably cumulative values; in any case they are qualified as being approximate. Qualitatively, these predictions are in accordance with the observations made above, and invoke the same technologies as the other concept studies.

Finally, despite their promise, only two of the novel configurations meet their respective targets. Moreover, those that do (Northrop Grumman's N+2 flying wing and the SAI BWB) are among the poorer performers for fuel-burn. This again highlights the likely future conflict between noise and fuel-efficiency demands. 


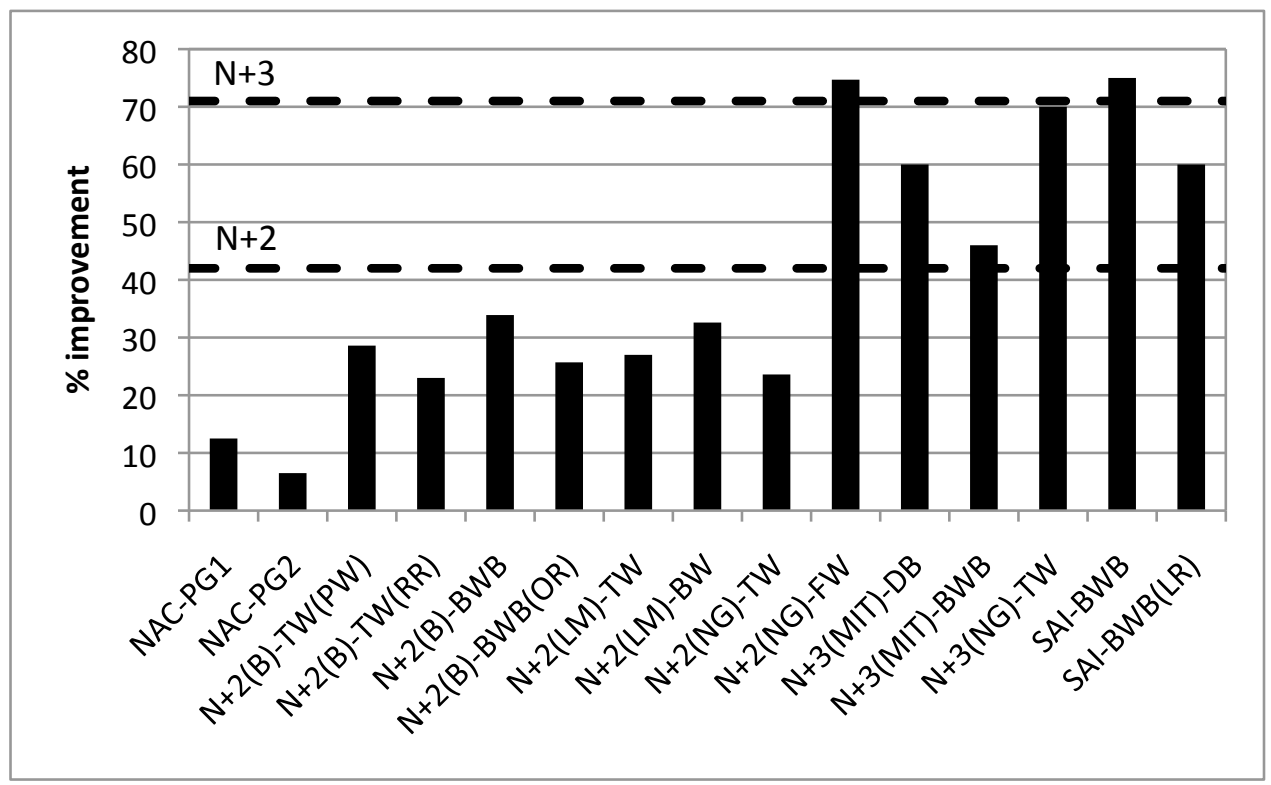

Figure 13 Noise reductions in cumulative EPNdB relative to Chapter 4 limits. NACRE study: PG1, turbofan TW with forward sweep; PG2, open-rotor version of PG1. NASA ERA N+2 studies: (B)-TW, Boeing TW with geared (PW) or conventional (RR) turbofans; (B)-BWB, Boeing BWB with geared turbofans or open rotors (OR); (LM)-TW, Lockheed Martin TW with turbofans; (LM)-BW, Lockheed Martin box-wing with geared turbofans; (NG)-TW, Northrop Grumman TW with turbofans; (NG)-FW, Northrop Grumman flying wing with embedded turbofans. NASA N+3 studies: (MIT)-DB, doublebubble with integrated turbofans; (MIT)-BWB, BWB with embedded, distributed propulsion; (NG)TW, Northrop Grumman TW with turbofans. Silent Aircraft Initiative: noise-optimised BWB with embedded or conventional (LR) turbofans. Dashed lines show NASA N+2 and N+3 targets.

\section{Discussion}

This section is split into two parts. In the first, we present our assessment of the studies described here, and their implications. In the second we move from the question of what could happen to what, in our opinion, should happen. The views expressed here, albeit inevitably personal and partial, were refined during TOSCA project discussions with industry experts, whose generosity with their valuable background knowledge we gratefully acknowledge.

\subsection{Critical assessment}

This review considers predictions for three key aircraft pollutants: $\mathrm{CO}_{2}$, NOx and noise. Among these, NOx is the most straightforward; there appears to be a broad consensus that significant future reductions are feasible, and on how these will be achieved. Furthermore, the required developments are essentially independent of the aircraft configuration. For these reasons, the discussion will be restricted to the issues of fuel consumption and noise.

For the former, the studies reviewed suggest that there is still scope for further development of the conventional, $\mathrm{TW}$, configuration. It is forecast that aerodynamic and propulsion system efficiencies will continue to improve, and that the ongoing shift from aluminium to advanced composite construction will yield significant weight gains. Depending on time-frame and mission, these 'business-as-usual' improvements are predicted to reduce the fuel consumption of new aircraft, relative to their current counterparts, by anything between $21 \%$ and $44 \%$ over the next forty years. These figures, however, should be viewed in the light of Fig. 1, whose extrapolation would paint a less optimistic picture. Only the use of composite materials represents a significant departure from such an extrapolation, and their potential for weight reduction is still anticipated, on the basis of simplified analyses, rather than proven in practice. Even if it exists to the extent hoped for, several aircraft generations may be required in order for designers to learn how to exploit it to the full.

Turning to more radical proposals, some form of laminar flow control is widely advocated. Here the potential is proven and accepted; the issues are operational. This technology places extremely stringent requirements on surface finish, which, even if attainable on a production aircraft, may not be maintainable in day-to-day flying. When suction is employed, blockage of the microscopic holes in the aircraft skin is an additional worry. Such problems raise a concern over reliability that must be addressed before the technology can be employed on a new aircraft design. The prospect that it might be implemented at the $\mathrm{N}+2$ horizon (2025) thus seems remote; even for the $\mathrm{N}+3$ timescale (2030-35), one would require long-term demonstrator work to start within the next few 
years. Furthermore, assuming reliable operation were shown to be feasible, it is still not certain that the limited benefits of partial laminarisation would justify the effort required; it might only be worthwhile for the greater prize of full laminarisation (cf. GBD's LFW configuration).

Less contentious from an operability viewpoint is open-rotor propulsion. Here there is no question that the technology is practically feasible, nor that it delivers efficiency benefits. It may, however, require acceptance of lower cruising speeds, and this may be why it has been proposed more frequently by European studies (current Airbus aircraft already cruise slightly slower than their Boeing counterparts). It is also, as mentioned previously, noisier than turbofan propulsion. Thus its future adoption, while not entailing an increase over current noise levels, would compromise further reductions in favour of improved fuel-burn.

The remaining radical TW technologies - aeroelastic wing, automatic stability and hybrid-electric power were suggested by one or two studies only. (Here we include the CRC tailless aircraft in the TW category.) Of these, automatic stability is well established and flight proven in military aircraft. However, it would take a huge shift in regulatory philosophy to accept a passenger aircraft that was naturally unstable, and hence unflyable, in the absence of its control system. The aeroelastic wing is proven at military demonstrator level; here the concerns over the need for automatic control are augmented by the issues of reliability and longevity (due to possible structural fatigue). Hybrid-electric power, on the other hand, is not only unproven; it is, at present, simply a means of displacing $\mathrm{CO}_{2}$ emissions elsewhere. Thus we doubt that any of these alternatives will gain acceptance in the first half of this century.

Moving on to novel configurations, several — the LFW, NACRE's PG2 and CRC's tailless aircraft - have already been considered. Of these, only the LFW represents a truly radical departure from the TW type; if achievable, it apparently holds great promise. Among the remainder, the BWB and the MIT double-bubble concepts are expected to be superior to their TW contemporaries, although even this statement might be considered contentious for the BWB; in Boeing's direct comparison for the N+2 designs, it is slightly worse. Noise is where the BWB really comes into its own; here it (and the similar flying-wing configuration) are only rivalled by the double-bubble. This is a crucial difference compared to fuel consumption. Whereas it is possible to envisage (given acceptance of some radical technologies) a TW-like aircraft that approaches the NASA N+3 and Flightpath 2050 fuel-burn goals, we very much doubt that the noise targets are remotely achievable without a radical shift away from the conventional configuration. Furthermore, even given this shift, fuel efficiency will almost certainly need to be compromised in order to reach them (witness the poorer performance of the noisetargeting SAI design compared to the versions with balanced objectives, and the preference for less-efficient turbofans over open-rotor propulsion when noise is at a premium).

To sum up, we claim that the predictions presented in this review should be viewed with a degree of scepticism. While the technologies invoked may indeed be potentially applicable, in many cases we have serious doubts over the practical possibility of their implementation, in concert, over the timescales suggested. By implication, therefore, we also question the NASA N+3 and Flightpath 2050 targets. In particular, we view the fuel-burn and noise goals as highly challenging in their own right; given the areas of potential mutual conflict, we believe them to be unrealistic taken together. Policymakers should not be seduced by the past gains shown in Figs. 1 and 3 ; these were achieved via a single, straightforward, technological change — increased engine BPR — that benefitted fuel-burn and noise simultaneously. Future gains will require much greater effort, and will involve difficult decisions between environmental and acoustic priorities.

\subsection{The way forward}

Assuming that oil prices remain at least around current levels of roughly US\$ 100 per barrel, there will be strong commercial pressure to reduce fuel consumption further. Meanwhile, regulatory constraints on noise and local pollutant emissions will continue to become more stringent. Evolutionary developments to address these demands will thus be a business necessity for both airframe and engine manufacturers. On this basis, continued reductions in fuel-burn, noise, and NOx can be expected in the future, but they are likely to fall well short of the various targets put forward by ACARE and NASA.

Of the more radical options, the open-rotor engine appears the most feasible, and offers a significant efficiency benefit (estimated fuel savings of up to $45 \%$ relative to baseline). However, despite the preliminary development work already undertaken, such a project would represent a huge financial risk for an engine manufacturer to shoulder alone, particularly given the associated noise concerns. In the authors' opinion, some form of government support will be necessary to realise an operational design.

Similar comments apply to the distortion-tolerant engine required for the MIT 'double-bubble' design. In addition, it requires a novel airframe configuration (albeit one that is only reliant on standard technologies). Despite these caveats, the concept offers a balance between risk and reward which is arguably the best of those presented here, especially for noise reduction. Unfortunately, given the development risks inherent even in a conventional new design (witness the troubled geneses of the Airbus A380 and the Boeing 787), it seems unlikely that an airframe company would elect to pursue it alone. 
Finally, all the remaining concepts - either additional TW technologies or novel configurations — are not yet at a stage where one could envisage launching a full-scale development program. Small-scale demonstrator projects are needed to investigate whether the potential that exists on paper is realisable in practice, and to identify the as-yet unforeseen difficulties that will inevitably crop up. Again, it seems highly unlikely that such projects would, or could, be undertaken by a commercial company alone.

In summary, then, it is likely that governments would have to bear at least part of the cost of any technology developments beyond those labelled here as 'evolutionary'. Whether or not they should do so is a question beyond our scope. However, we can say with certainty that new technology should not be regarded as the only source of solutions to the aircraft emissions problem, as the following points illustrate.

The relative inefficiency of aircraft over flight distances much less than their design range has already been noted, along with the inherent wastefulness of long-range designs. However, aircraft with ranges lower than the narrow-body turbofan type already exist: turboprops. If used instead of narrow-bodies on all flights under 1,000 $\mathrm{km}(540 \mathrm{nmi})$, they would achieve fuel savings of 13\% and upwards, with relatively small travel-time penalties. Unfortunately, passenger acceptance issues (both real and perceived) form a barrier to their uptake. More generally, narrow-body aircraft with reduced design range and cruising speed low enough to allow unswept wings would deliver substantial benefits even at today's technology levels. They do not currently exist because airlines do not demand them; the economic value of speed and the flexibility afforded by excess range capability take priority.

Finally, as for most transport modes, demand growth is perhaps the single biggest issue for aviation emissions. Only small differences in annual growth rate are needed to generate a range of values for total fuel consumption in 25 years time that easily covers the difference between the least and most optimistic estimates of possible technological benefits. Unpalatable as it may be, some form of intervention above and beyond technology policy is likely to be required in order to control emission levels.

None of the operational and behavioural issues touched on here is straightforward to address. Equally, though, all are amenable to improvement via alterations to current economic incentives. Whether the political will to implement such alterations exists is an open question. What is certain, in our view, is that even the most optimistic estimates of future technology benefits are in no way great enough to provide a legitimate excuse for inaction on other fronts.

\section{Conclusions}

In this paper, we have discussed the potential contribution of future technology to reducing the levels of three key aircraft emissions: carbon dioxide, oxides of nitrogen, and noise. Aerosols, the other significant concern, have not been addressed, on the basis that their effect is currently too uncertain to warrant its influencing design decisions. Consideration has also been limited to the aircraft itself; air-traffic-control-related measures and alternative fuels have been excluded as they are covered in companion papers (Hileman, Reynolds, this issue). The discussion is based on a review of the future-concept studies that have appeared over the past decade.

These studies have been carried out in the context of continued demand for noise and local air quality improvements, coupled with the more recent recognition of carbon dioxide as a pollutant, via its greenhouse-gas property. European (ACARE) and American (NASA) aviation organisations have responded to these concerns by setting future targets on all three emissions. Broadly, these propose further proportional reductions of extent similar to those already achieved in the fifty-five years since the start of the jet-transport age, within forty years or fewer, and subject to the law of diminishing returns. Thus, purely on a historical basis, they will require radical developments. This view is supported by the studies reviewed here; even the most optimistic of the predictions for 'business-as-usual' progress fall well short, despite positing improvements significantly over and above those that can be expected from an extrapolation of past trends.

Of the three targets, that for NOx is generally agreed to be the most achievable; it also has the advantage that it is to some extent independent of both the aircraft configuration and the other two emissions, reducing the potential for conflict. Noise and $\mathrm{CO}_{2}$ (or, equivalently, fuel-burn) appear to represent greater challenges. The fuel-burn reduction targets may be at least approachable with recognisable derivatives of the conventional configuration if one is prepared to invoke an appropriate combination of radical technologies from the following list: counterrotating propellor engines, laminar flow control, automatic stability control, aeroelastic wings, double-bubble lifting fuselage, box-wing construction. However, it appears certain that the noise goals will only be achievable with radical, flying-wing-type designs. Furthermore, compromises on fuel efficiency will be necessary to achieve optimum noise reduction.

To sum up, the fundamental conclusion of the work reviewed here is that the longer-term ACARE and NASA goals cannot be achieved in concert. Therefore, given the tendency of paper studies towards optimism, and the unforeseen obstacles that inevitably arise in the practical implementation of novel technologies, we regard these goals as unrealistic. 
The adoption of unrealistic future targets may be justifiable on the basis that they provide a stimulus for advances that would not otherwise have been achieved. However, there is a danger that they distort the policy debate by implying that technological improvements are capable of solving the problem without recourse to operational or behavioural change. In our view, this is simply not the case.

If the need for such change is accepted, then existing technology can already provide significant benefits, on the fuel-burn front at least. We should fly slower, using propellor engines if possible, and with aircraft suited to the mission range. In particular, far greater use of turboprop aircraft should be encouraged.

This is not to say that future technological development is futile; indeed, the gap between today's aircraft and the long-term ACARE/NASA goals is so large that failure to meet them could still represent very significant improvement. However, the risks inherent in any departure from evolutionary refinement of the conventional configuration are too great to be borne by industry alone; society, via governmental support, will be required to contribute. Whether the associated cost can be justified is a matter for future debate. Similarly, the conflict between noise and fuel-burn reduction means that difficult choices over their respective priorities will have to be made. There is, unfortunately, no silver bullet.

\section{Acknowledgments}

The authors are grateful to the following for their help in granting and/or obtaining copyright permissions: Ed Greitzer, Kenneth Martin, Linda Nicol, Sophie Schlingemann. 
Appendix: Concept Study Summary Tables

Table A.1 Aircraft concepts

\begin{tabular}{|c|c|c|c|}
\hline Study & EIS & Description $^{a}$ & Identifier \\
\hline \multirow[t]{11}{*}{ Greener by Design } & \multirow[t]{11}{*}{2050} & Long-range TW (SW-LR 2050) & GBD-TWL \\
\hline & & Medium-range TW (SW-MR 2050) & GBD-TWM \\
\hline & & Long-range TW with ICR engine (SW-LR ICR-2050) & GBD-TWL(ICR) \\
\hline & & Medium-range TW with ICR engine (SW-MR ICR-2050) & GBD-TWM(ICR) \\
\hline & & $\begin{array}{l}\text { Medium-range TW with HLFC on fin, tail and nacelle } \\
\text { (SW-MR: HLFC-FTN 2050) }\end{array}$ & GBD-TWM(HLFa) \\
\hline & & $\begin{array}{l}\text { Medium-range TW with HLFC on fin, tail, nacelle and } \\
\text { wing (SW-MR: HLFC-FTNW 2050) }\end{array}$ & GBD-TWM(HLFb) \\
\hline & & $\begin{array}{l}\text { Medium-range TW with ICR engine and HLFC on fin, tail, } \\
\text { nacelle and wing (SW-MR: HLFC-FTNW ICR-2050) }\end{array}$ & GBD-TWM(MAX) \\
\hline & & BWB with turbofan engines (BWB 2050) & GBD-BWB \\
\hline & & BWB with open-rotor engines (BWB-UDF 2050) & GBD-BWB(OR) \\
\hline & & LFW with turbofan engines (LFW 2050) & GBD-LFW \\
\hline & & LFW with open-rotor engines (LFW-UDF 2050) & GBD-LFW(OR) \\
\hline \multirow[t]{3}{*}{ NACRE } & \multirow[t]{3}{*}{2015} & TW with tail-mounted turbofans and forward sweep (PG1) & NAC-PG1 \\
\hline & & Open-rotor-powered version of PG1 (PG2) & NAC-PG2 \\
\hline & & BWB with turbofan engines (FW2) & NAC-BWB \\
\hline \multirow{4}{*}{$\begin{array}{l}\text { NASA ERA N+2: } \\
\text { Boeing }\end{array}$} & \multirow[t]{4}{*}{2025} & TW with geared turbofans (0005) & $\mathrm{N}+2(\mathrm{~B})-\mathrm{TW}(\mathrm{PW})$ \\
\hline & & TW with advanced turbofans (0007) & $N+2(B)-T W(R R)$ \\
\hline & & BWB with geared turbofans (009A) & $\mathrm{N}+2(\mathrm{~B})-\mathrm{BWB}$ \\
\hline & & BWB with open rotors (0013) & $N+2(B)-B W B(O R)$ \\
\hline \multirow{2}{*}{$\begin{array}{l}\text { NASA ERA N+2: } \\
\text { Lockheed Martin }\end{array}$} & \multirow[t]{2}{*}{2025} & TW with turbofans (conventional) & $\mathrm{N}+2(\mathrm{LM})-\mathrm{TW}$ \\
\hline & & Box-wing with geared turbofans (PSC) & $N+2(L M)-B W$ \\
\hline \multirow{2}{*}{$\begin{array}{l}\text { NASA ERA N+2: } \\
\text { Northrop Grumman }\end{array}$} & \multirow[t]{2}{*}{2025} & TW with turbofans (baseline) & $N+2(N G)-T W$ \\
\hline & & Flying wing with embedded turbofans (PSC) & $N+2(N G)-F W$ \\
\hline \multirow[t]{4}{*}{ NASA N+3: Boeing } & \multirow[t]{4}{*}{2030} & TW with turbofans (Refined SUGAR) & $N+3(B)-T W$ \\
\hline & & TW: braced wing with turbofans (SUGAR High) & $N+3(B)-B W$ \\
\hline & & Hybrid electric version of SUGAR High (SUGAR Volt) & $\mathrm{N}+3(\mathrm{~B})-\mathrm{BW}(\mathrm{HE})$ \\
\hline & & BWB with turbofans (SUGAR Ray) & $\mathrm{N}+3(\mathrm{~B})-\mathrm{BWB}$ \\
\hline NASA N+3: CRC & 2035 & $\begin{array}{l}\text { Tailless TW with open rotors, advanced aeroelastic wing } \\
\text { and stability control }\end{array}$ & $\mathrm{N}+3(\mathrm{CRC})-\mathrm{TTW}$ \\
\hline \multirow[t]{2}{*}{ NASA N+3: MIT } & \multirow[t]{2}{*}{2035} & Double-bubble with integrated turbofans (D8.5) & $\mathrm{N}+3(\mathrm{MIT})-\mathrm{DB}$ \\
\hline & & BWB with embedded, distributed propulsion $(\mathrm{H} 3.2)$ & $\mathrm{N}+3(\mathrm{MIT})-\mathrm{BWB}$ \\
\hline $\begin{array}{l}\text { NASA N+3: } \\
\text { Northrop Grumman }\end{array}$ & $\begin{array}{l}2030- \\
2035\end{array}$ & TW with turbofans (SELECT) & $\mathrm{N}+3(\mathrm{NG})-\mathrm{TW}$ \\
\hline \multirow{2}{*}{$\begin{array}{l}\text { Silent Aircraft } \\
\text { Initiative }\end{array}$} & \multirow[t]{2}{*}{2025} & BWB with embedded turbofans (SAX-40) & SAI-BWB \\
\hline & & BWB with turbofans (SAX-L/R1) & SAI-BWB(LR) \\
\hline \multirow[t]{4}{*}{ TOSCA } & \multirow[t]{4}{*}{2025} & TW with turbofans (narrow-body replacement) & TOS-TF \\
\hline & & TW with open rotors (fast open rotor) & TOS-FOR \\
\hline & & Lower speed open-rotor TW (reduced-speed open rotor) & TOS-RSOR \\
\hline & & TW with turboprop engines (turboprop replacement) & TOS-TP \\
\hline
\end{tabular}

a) Concept references used by the teams producing the studies are given in brackets. 
Table A.2 Mission parameters for the concept aircraft

\begin{tabular}{|c|c|c|c|c|c|}
\hline Identifier & Gross weight (lb) & Passengers & Range (nmi) & Cruise Mach No. & Cruise altitude (ft) \\
\hline GBD-TWL & $N / A^{a}$ & $N / A^{a}$ & 8094 & 0.85 & 40000 \\
\hline GBD-TWM & $N / A^{a}$ & $N / A^{a}$ & 2698 & 0.85 & 32000 \\
\hline GBD-TWL(ICR) & $N / A^{a}$ & $N / A^{a}$ & 8094 & 0.85 & 40000 \\
\hline GBD-TWM(ICR) & $N / A^{a}$ & $N / A^{a}$ & 2698 & 0.85 & 32000 \\
\hline GBD-TWM(HLFa) & $N / A^{a}$ & $N / A^{a}$ & 2698 & 0.85 & 32000 \\
\hline GBD-TWM(HLFb) & $N / A^{a}$ & $N / A^{a}$ & 2698 & 0.85 & 32000 \\
\hline GBD-TWM(MAX) & $N / A^{a}$ & $N / A^{a}$ & 2698 & 0.85 & 32000 \\
\hline GBD-BWB & $N / A^{a}$ & $N / A^{a}$ & 2698 & 0.85 & 32000 \\
\hline GBD-BWB(OR) & $N / A^{a}$ & $N / A^{a}$ & 2698 & 0.8 & 32000 \\
\hline GBD-LFW & $N / A^{a}$ & $N / A^{a}$ & 4856 & 0.85 & 29000 \\
\hline GBD-LFW(OR) & $N / A^{a}$ & $N / A^{a}$ & 4856 & 0.8 & 29000 \\
\hline NAC-PG1 & $?$ & $?$ & $?$ & 0.76 & $?$ \\
\hline NAC-PG2 & $?$ & $?$ & $?$ & 0.74 & $?$ \\
\hline NAC-BWB & $1,388,900$ & 750 & 7650 & $?$ & $?$ \\
\hline$N+2(B)-T W(P W)$ & $?$ & 224 & 8000 & 0.85 & $?$ \\
\hline$N+2(B)-T W(R R)$ & $?$ & 224 & 8000 & 0.85 & $?$ \\
\hline$N+2(B)-B W B$ & $?$ & 224 & 8000 & 0.85 & $?$ \\
\hline$N+2(B)-B W B(O R)$ & $?$ & 224 & 8000 & 0.85 & $?$ \\
\hline $\mathrm{N}+2(\mathrm{LM})-\mathrm{TW}$ & 364,500 & 224 & 8000 & 0.85 & up to 45000 \\
\hline $\mathrm{N}+2(\mathrm{LM})-\mathrm{BW}$ & 365,900 & 224 & 8000 & 0.85 & up to 47000 \\
\hline$N+2(N G)-T W$ & 466,000 & 224 & 8000 & 0.85 & $43000^{b}$ \\
\hline$N+2(N G)-F W$ & 427,100 & 224 & 8000 & 0.85 & $51000^{b}$ \\
\hline$N+3(B)-T W$ & 139,700 & 154 & 3500 & 0.7 & 38400 \\
\hline $\mathrm{N}+3(\mathrm{~B})-\mathrm{BW}$ & 176,800 & 154 & 3500 & 0.7 & 42100 \\
\hline $\mathrm{N}+3(\mathrm{~B})-\mathrm{BW}(\mathrm{HE})$ & 154,900 & 154 & 3500 & 0.7 & 42000 \\
\hline$N+3(B)-B W B$ & 172,600 & 154 & 3500 & 0.7 & 40800 \\
\hline $\mathrm{N}+3(\mathrm{CRC})-\mathrm{TTW}$ & 127,200 & 180 & 2774 & 0.71 & 34000 \\
\hline $\mathrm{N}+3(\mathrm{MIT})-\mathrm{DB}$ & 101,600 & 180 & 3000 & 0.74 & $45500^{b}$ \\
\hline $\mathrm{N}+3(\mathrm{MIT})-\mathrm{BWB}$ & 470,600 & 354 & 7600 & 0.83 & $37900^{b}$ \\
\hline$N+3(N G)-T W$ & 80,500 & 120 & 1600 & 0.75 & 45000 \\
\hline SAI-BWB & 332,600 & 215 & 5000 & 0.8 & $42500^{b}$ \\
\hline SAI-BWB(LR) & 347,900 & 215 & 5000 & 0.8 & $42500^{b}$ \\
\hline TOS-TF & 162,000 & 179 & 2700 & 0.77 & 38000 \\
\hline TOS-FOR & 162,000 & 179 & 2700 & 0.77 & 40000 \\
\hline TOS-RSOR & 162,000 & 179 & 2700 & 0.66 & 40000 \\
\hline TOS-TP & 49,600 & 74 & 810 & 0.4 & 22000 \\
\hline
\end{tabular}

a) The Greener-by-Design analysis is in terms of weight ratios (relative to the total); hence absolute weights and passenger numbers are not available.

b) For cases where height is gained during cruise, the average altitude is given. 
Table A.3 Fuel-burn-reducing technologies invoked by the concept studies

\begin{tabular}{|c|c|c|c|c|c|c|c|c|c|c|c|c|c|c|c|}
\hline Identifier & 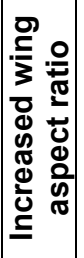 & $\frac{\text { 岁 }}{\mathbf{z}}$ & $\begin{array}{l}\text { U } \\
\text { 폰 }\end{array}$ & $\frac{\frac{n}{0}}{\frac{0}{\alpha}}$ & 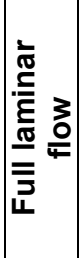 & 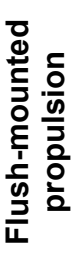 & 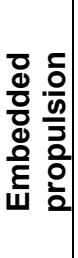 & 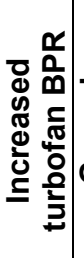 & 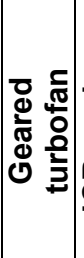 & 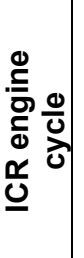 & 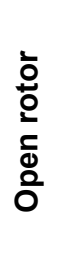 & 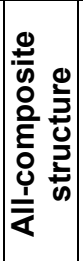 & 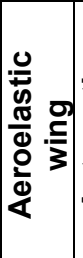 & 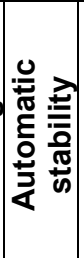 & 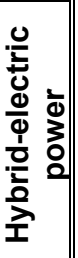 \\
\hline GBD-TWL & & & & & & & & & & & & $\mathrm{X}$ & & & \\
\hline GBD-TWM & & & & & & & & & & & & $\mathrm{X}$ & & & \\
\hline GBD-TWL(ICR) & & & & & & & & & & $X$ & & $X$ & & & \\
\hline GBD-TWM(ICR) & & & & & & & & & & $X$ & & $X$ & & & \\
\hline GBD-TWM(HLFa) & & & $X$ & & & & & & & & & $X$ & & & \\
\hline GBD-TWM(HLFb) & & & $\mathrm{X}$ & & & & & & & & & $\mathrm{X}$ & & & \\
\hline GBD-TWM(MAX) & & & $\mathrm{X}$ & & & & & & & 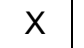 & & $\mathrm{X}$ & & & \\
\hline GBD-BWB & & & & & & & & & & & & $X$ & & & \\
\hline GBD-BWB(OR) & & & & & & & & & & & $X$ & $X$ & & & \\
\hline GBD-LFW & & & & & $\mathrm{X}$ & & & & & & & $\mathrm{X}$ & & & \\
\hline GBD-LFW(OR) & & & & & $\mathrm{X}$ & & & & & & $\mathrm{X}$ & $X$ & & & \\
\hline NAC-PG1 & $X$ & $X$ & & & & & & & & & & & & & \\
\hline NAC-PG2 & $X$ & $X$ & & & & & & & & & $X$ & & & & \\
\hline NAC-BWB & & & & & & $\mathrm{X}$ & & & & & & & & & \\
\hline $\mathrm{N}+2(\mathrm{~B})-\mathrm{TW}(\mathrm{PW})$ & $X$ & & $X$ & $X$ & & & & $X$ & $\mathrm{X}$ & & & $X$ & & & \\
\hline$N+2(B)-T W(R R)$ & $\mathrm{X}$ & & $\mathrm{X}$ & $\mathrm{X}$ & & & & $\mathrm{X}$ & & & & $\mathrm{X}$ & & & \\
\hline$N+2(B)-B W B$ & & & $\mathrm{X}$ & $\mathrm{X}$ & & & & $X$ & $\mathrm{X}$ & & & $\mathrm{X}$ & & & \\
\hline $\mathrm{N}+2(\mathrm{~B})-\mathrm{BWB}(\mathrm{OR})$ & & & $X$ & $X$ & & & & & & & $X$ & $X$ & & & \\
\hline$N+2(L M)-T W$ & $X$ & $x^{a}$ & $x^{a}$ & & & & & $X$ & & & & $X$ & & & \\
\hline$N+2(L M)-B W$ & $X$ & $X^{a}$ & $X^{a}$ & & & & & $X$ & $X$ & & & $X$ & & & \\
\hline$N+2(N G)-T W$ & & $x^{a}$ & $x^{a}$ & $X$ & & & & $?^{\mathrm{b}}$ & & & & $?^{\mathrm{b}}$ & & & \\
\hline$N+2(N G)-F W$ & & $X^{a}$ & $x^{a}$ & $X$ & & & $X$ & $X$ & & & & $X$ & & & \\
\hline $\mathrm{N}+3(\mathrm{~B})-\mathrm{TW}$ & $X$ & $X^{a}$ & $x^{a}$ & $X$ & & & & $X$ & & & & $X$ & & $x$ & \\
\hline $\mathrm{N}+3(\mathrm{~B})-\mathrm{BW}$ & $x$ & $X^{a}$ & $x^{a}$ & $X$ & & & & $X$ & & & & $X$ & & $X$ & \\
\hline $\mathrm{N}+3(\mathrm{~B})-\mathrm{BW}(\mathrm{HE})$ & $X$ & $X^{a}$ & $x^{a}$ & $X$ & & & & $X$ & & & & $\mathrm{X}$ & & $X$ & $\mathrm{X}$ \\
\hline $\mathrm{N}+3(\mathrm{~B})-\mathrm{BWB}$ & & $X^{a}$ & $X^{a}$ & $X$ & & & & $X$ & & & & $X$ & & $X$ & \\
\hline $\mathrm{N}+3(\mathrm{CRC})-\mathrm{TTW}$ & $X$ & $X^{a}$ & $x^{a}$ & & & & & & & & $X$ & $X$ & $X$ & $X$ & \\
\hline $\mathrm{N}+3(\mathrm{MIT})-\mathrm{DB}$ & $x$ & $x$ & & & & $x$ & & $X$ & & & & $X$ & & & \\
\hline N+3(MIT)-BWB & & & & & & & $X$ & $X$ & & & & $X$ & & & \\
\hline$N+3(N G)-T W$ & $\mathrm{X}$ & $\mathrm{X}$ & & & & & & $\mathrm{X}$ & & $x$ & & $\mathrm{X}$ & $\mathrm{X}$ & & \\
\hline SAI-BWB & & & & & & & $X$ & $\mathrm{X}$ & & & & $X$ & & & \\
\hline SAI-BWB(LR) & & & & & & & & $X$ & & & & $X$ & & & \\
\hline TOS-TF & $X$ & & & & & & & $X$ & & & & $X$ & & & \\
\hline TOS-FOR & $X$ & & & & & & & & & & $X$ & $X$ & & & \\
\hline TOS-RSOR & $X$ & & & & & & & & & & $X$ & $\mathrm{X}$ & & & \\
\hline TOS-TP & & & & & & & & & & & & & & & \\
\hline
\end{tabular}

a) Studies that invoke laminar flow without further specification have both 'NLF' and 'HLFC' checked.

b) '?' indicates that the technology is presumably invoked, but is not explicitly cited. 
Table A.4 Noise-reducing technologies invoked by the concept studies

\begin{tabular}{|c|c|c|c|c|c|}
\hline Identifier & Jet noise & Blade noise & $\begin{array}{l}\text { Landing } \\
\text { gear }\end{array}$ & $\begin{array}{l}\text { Leading-edge } \\
\text { slats }\end{array}$ & $\begin{array}{l}\text { Trailing-edge } \\
\text { flaps }\end{array}$ \\
\hline NAC-PG1 & & Contra-rotating fan ${ }^{a}$ & & Substituted & \\
\hline NAC-PG2 & Open rotor & a & & Substituted & \\
\hline$N+2(B)-T W(P W)$ & Higher BPR & Advanced liners & Fairings? & Substituted & \\
\hline$N+2(B)-T W(R R)$ & Higher BPR & Advanced liners? & Fairings? & Substituted & \\
\hline$N+2(B)-B W B$ & Higher BPR & $\begin{array}{l}\text { Advanced liners, } \\
\text { shielding }\end{array}$ & Fairings? & Substituted & Substituted \\
\hline $\mathrm{N}+2(\mathrm{~B})-\mathrm{BWB}(\mathrm{OR})$ & Open rotor & Shielding & Fairings? & Substituted & Substituted \\
\hline$N+2(L M)-T W$ & Higher BPR & & Fairings & Treated & Treated \\
\hline$N+2(L M)-B W$ & Higher BPR & & Fairings & Treated & Treated \\
\hline$N+2(N G)-T W$ & Higher BPR? & & & & \\
\hline $\mathrm{N}+2(\mathrm{NG})-\mathrm{FW}$ & Higher BPR & Embedding, shielding & & & \\
\hline $\mathrm{N}+3(\mathrm{~B})-\mathrm{TW}$ & Higher BPR & Advanced liners & Fairings & Substituted & Treated \\
\hline $\mathrm{N}+3(\mathrm{~B})-\mathrm{BW}$ & Higher BPR & Advanced liners & Fairings & Substituted & Treated \\
\hline $\mathrm{N}+3(\mathrm{~B})-\mathrm{BW}(\mathrm{HE})$ & Higher BPR & Advanced liners & Fairings & Substituted & Treated \\
\hline$N+3(B)-B W B$ & Higher BPR & $\begin{array}{l}\text { Advanced liners, } \\
\text { shielding }\end{array}$ & Fairings & Substituted & Treated \\
\hline $\mathrm{N}+3(\mathrm{MIT})-\mathrm{DB}$ & Higher BPR & $\begin{array}{l}\text { Rearward liners, } \\
\text { shielding }\end{array}$ & Fairings & Eliminated & \\
\hline $\mathrm{N}+3(\mathrm{MIT})-\mathrm{BWB}$ & Higher BPR & Embedding, shielding & Fairings & Substituted & Eliminated \\
\hline$N+3(N G)-T W$ & Higher BPR & Advanced liners & Fairings & Eliminated & \\
\hline SAI-BWB & Higher BPR & Embedding, shielding & Fairings & Substituted & Eliminated \\
\hline SAI-BWB(LR) & Higher BPR & Shielding & Fairings & Substituted & Eliminated \\
\hline
\end{tabular}

a) Shielding is not included for the NACRE concepts, because it was found to be almost negligible. 


\section{References}

Bonet, J.T. (2012) 'Boeing ERA N+2 advanced vehicle concept results'. Oral presentation at the $50^{\text {th }}$ AIAA Aerospace Sciences Meeting; https://www.aiaa.org/uploadedFiles/About-AIAA/Press-Room/Key_SpeechesReports-and-Presentations/2012/Bonet-John-Boeing-AVC-AIAA-GEPC2.pdf.

Bradley, M.K. and Droney, C.K. (2011) 'Subsonic ultra green aircraft research: Phase I final report'. NASA/CR-2011-216847.

Bruner, S. and 7 others (2010) 'NASA N+3 subsonic fixed wing silent efficient low-emissions commercial transport (SELECT) vehicle study'. NASA/CR-2010-216798.

Collier, F.S. (2012) 'NASA aeronautics Environmentally Responsible Aviation project'. Oral presentation at the $50^{\text {th }}$ AIAA Aerospace Sciences Meeting; https://www.aiaa.org/uploadedFiles/About-AIAA/PressRoom/Key_Speeches-Reports-and-Presentations/2012/Collier-NASA-AVC-AIAA-GEPC2-2.pdf.

DGAC (2012) 'Noise certification database'. Online database hosted at http://noisedb.stac.aviationcivile.gouv.fr.

Dowling, A.P. (2007) 'The Silent Aircraft Initiative - overview'. AIAA-2007-0452, presented at 45th AIAA Aerospace Sciences Meeting and Exhibit.

Drake, A. (2012) 'NASA Environmentally Responsible Aviation (ERA) N+2 advanced vehicle study'. Oral presentation at the $50^{\text {th }}$ AIAA Aerospace Sciences Meeting; https://www.aiaa.org/uploadedFiles/AboutAIAA/Press-Room/Key_Speeches-Reports-and-Presentations/2012/Drake-Northrop-AVC-AIAAGEPC2.pdf.

European Commission (2001) European Aeronautics: A Vision for 2020, Office for Official Publications of the European Communities, Luxembourg, ISBN 92-894-0559-7.

European Commission (2011) Flightpath 2050: Europe's Vision for Aviation, Publications Office of the European Union, Luxembourg, ISBN 978-92-79-19724-6, doi 10.2777/50266.

Frota, J. and 15 others (2011) 'NACRE final activity report 2005-2010'. NACRE project report; http://www.transportresearch.info/Upload/Documents/201210/20121030_161035_66969_Final_Activity_Report_R1.0.pdf.

Green, J.E. (2002) 'Greener by Design - the technology challenge', Aeronautical Journal, 106, pp. 57-113.

Greitzer, E.M. and 25 others (2011) 'N+3 aircraft concept designs and trade studies, final report'. NASA/CR2011-216794.

Hall, C.A. (2009) 'Low noise engine design for the Silent Aircraft Initiative', Aeronautical Journal, 113, pp. 599-607.

Hall, C.A., Schwartz, E. and Hileman, J.I. (2009) 'Assessment of technologies for the Silent Aircraft Initiative', AIAA Journal of Propulsion and Power, 25(6), pp. 1153-1162.

Henderson, S.C. and Wickrama, U.K. (1999) Figure 9-3 of 'Aircraft emissions: current inventories and future scenarios', in Penner, J.E., Lister, D.H., Griggs, D.J., Dokken, D.J. and McFarland, M. (eds) (1999) Aviation and the Global Atmosphere: a Special Report of IPCC Working Groups I and III, Cambridge University Press, Cambridge.

ICAO (2012) 'Emissions databank'. Online database hosted at http://easa.europa.eu/environment/edb/aircraftengine-emissions.php.

Lee J. J., Lukachko S.P., Waitz I.A. and Schäfer A. (2001) 'Historical and future trends in aircraft performance, cost and emissions', Annual Reviews in Energy and the Environment, 26, pp. 167-200.

Martin, K.C. 'NASA Environmentally Responsible Aviation'. Oral presentation at the $50^{\text {th }}$ AIAA Aerospace Sciences Meeting; https://www.aiaa.org/uploadedFiles/About-AIAA/Press-Room/Key_Speeches-Reportsand-Presentations/2012/Martin-Lockheed-AVC-AIAA-GEPC2.pdf. 
Prather, M. and Sausen, R. (1999) 'Potential climate change from aviation', in Penner, J.E., Lister, D.H., Griggs, D.J., Dokken, D.J. and McFarland, M. (eds) (1999) Aviation and the Global Atmosphere: a Special Report of IPCC Working Groups I and III, Cambridge University Press, Cambridge.

Raymer, D.P. (2006) Aircraft Design: a Conceptual Approach, AIAA, Reston VA.

Raymer, D.P., Wilson, J., Perkins, H.D., Rizzi, A., Zhang, M. and Ramirez Puentes, A. (2011) 'Advanced technology subsonic transport study'. NASA/TM-2011-217130.

Schäfer A., Heywood J.B. and Weiss M.A. (2006) 'Future fuel cell and internal combustion engine automobile technologies: a 25 year life-cycle and fleet impact assessment', Energy-The International Journal, 31(12), pp. 1728-1751.

Torenbeek, E. (1997) 'Cruise performance and range predictions reconsidered', Prog. Aerosp. Sci, 35(5-6), pp. 285-321.

Vera Morales, M., Graham, W.R., Hall, C.A. and Schäfer, A. (2011) 'Techno-economic analysis of aircraft'. TOSCA project work-package 2 final report; http://www.toscaproject.org/FinalReports/TOSCA_WP2_Aircraft.pdf. 